\title{
Coupling of heterogeneous kinematics and Finite Element approximations applied to composite beam structures
}

\author{
C. Wenzel, P. Vidal *, M. D’Ottavio, O. Polit \\ Laboratoire Energétique Mécanique Electromagnétisme EA4416, Universit Paris Ouest - Nanterre La Défense, 50, rue de Sévres, 92410 Ville d'Avray, France
}

\begin{abstract}
A B S T R A C T
In the framework of the modeling of composite beam structures, the eXtended Variational Formulation (XVF) is carried out to couple different kinematics. The purpose is to take advantages of efficient models and reduce the overall computational cost without loss of local precision. In this way, the structure is divided into non-overlapping domains with different kinematics. Local domains of interest are described with advanced models, such as refined Sinus model, to precisely describe local behavior, while the remaining global domain uses simple classical models. Each of the kinematics needs a suitable Finite Element (FE) approximation, therefore the coupling of different FE approximations is also addressed. The present approach is assessed on homogeneous and sandwich structures. It is compared with classical Multiple Point Constraints vias penalty. The study has shown the need of the introduction of a new operator for the layered structures. The results are very promising.
\end{abstract}

\section{Introduction}

Composite structures are widely used in industry due to their excellent weight-specific mechanical properties. However, the price to be paid is a more complex behavior with respect to classical monolithic ones. In particular, a high computational effort is required for obtaining accurate stress fields due to material heterogeneity and anisotropy, even if their geometry often calls for the application of structural models of reduced dimensionality like beams (slender solids) and plate/shells (thin solids). Nevertheless, in many cases high stress gradients are limited to several local domains of interest, while large portions of the structure can effectively profit of the global geometric slenderness and/or thinness. In light of an optimization of the computational effort, it appears thus interesting to model these large portions of the structure by means of classical structural models, which rely on low-order kinematics assumptions such as Euler-Bernoulli's or Kirchhoff-Love's, and to employ a refined description for the local regions in which an adequate resolution of the stress gradients is required. Such globallocal analysis technique is well known, in which most often a solid (3D) model is used for the local domains [1]. Instead of using a full 3D model, in many cases it is however possible to employ structural models of lower dimensionality and refined, higher-order kinematics. This way, a further reduction of the computational

\footnotetext{
* Corresponding author. Tel.: +33 140974855.

E-mail address: philippe.vidal@u-paris10.fr (P. Vidal).
}

effort may be achieved without degrading the local accuracy in the framework of a global-local analysis method. Within this scope, the present work aims at developing a dedicated technique to couple beam Finite Elements formulated with heterogeneous kinematics, where a low-order classical kinematics is used in a global, "simple" sub-domain and a refined, higher-order one in a local, "complex" sub-domain.

First applied to the multiphysics environment, the coupling of different sub-domains has been a subject of several research activities from which different techniques are available today. These can take into account the coupling of domains of homogeneous dimensionality but heterogeneous kinematical description, or domains of heterogeneous dimensionality and homogeneous kinematical description. In the following, the commonly used techniques shall be classified following the topology of the arrangement of the domains: sub-domains with complete and partial overlap as well as non-overlapping sub-domains will be distinguished. Such a classification appears in fact more intuitive than a comparison including the question of homogeneous or heterogeneous dimensionality.

The complete overlap is first considered. Fish et al. [2,3] improved the accuracy of results by superimposing additional mesh of higher-order hierarchical elements in the region of interest. This method is called the superposition version of the Finite Element Method (FEM), also known as the s-Version of the FEM. Homogeneous boundary conditions along the common boundary are applied to keep the $\mathrm{C}^{0}$ continuity of the displacements. The displacement field in the local area is thus obtained as the sum of the 
higher order mesh and the global mesh. The solution for homogeneous dimensional problems is obtained directly with incompatible meshes. Reddy and Robbins [4] proposed to combine the variable kinematics Finite Elements with the s-Version approach. This represents an extension of Fish's method in that it couples Finite Elements with different mathematical model types. So, the local overlay mesh of variable kinematic elements can include both Equivalent Single Layer and LayerWise approaches. While both mentioned techniques resort to a simultaneous solution of the overlapping domains, Gendre et al. [5] developed an iterative method for domains with different description. Their two-scale analysis method for local sub-domains combines local and global contributions. The global domain is assumed to have linear elastic properties, which can be also homogenized, while material and geometric non-linearity can be included for the local domain. It uses a two-scale approximation of the Schur complement of the local domain's stiffness matrix. The use of a weighted combination of Dirichlet and Neumann boundary conditions on the local domain enables relatively low number of iterations and assures the convergence of the solution.

As far as the techniques with partial overlap are concerned, Ben Dhia et al. [6] proposed the so-called Arlequin method. Three regions are here considered: a domain using a simple kinematical model, a domain using a complex kinematical model and the sub-domain where both domains overlap. The connection in the superposition zone is performed in a weak sense via Lagrange Multipliers, and the total internal energy is partitioned between the overlapping models. The Arlequin method was systematically used by $\mathrm{Hu}$ and coworkers to analyze sandwich structures for both linear [7] and nonlinear applications [8,9]. In these applications, the Arlequin framework has been used to couple coarse and refined models, including both dimensionally homogeneous and heterogeneous models, as well as a FE model with a known solution. Biscani and coworkers continued this approach by referring to dimensionally homogeneous sub-domains with low-order and high-order kinematics for beams [10] and plates [11]. Note that the superposition volume of the Arlequin method can be degenerated to a surface coupling of the domains. However, some perturbations are induced close to the interface due to the coupling formulation between the domains.

Finally, non-overlapping techniques are widely used in open literature. The classical Lagrange Multipliers method allows to link the displacements between two adjacent subdomains by adding an interface constraint functional in the mechanical formulation of the problem. The interface potential was first proposed by Prager [12] to treat internal physical discontinuities by linking the unknowns of the sub-domains through a single Lagrange Multipliers field. This classical, two-fields formulation was used in [13] to combine variable kinematic models in the framework of the Carrera's Unified Formulation for beam structures. The mortar method is also mentioned, which provides the well-suited Lagrange Multipliers space [14]. The Lagrange Multipliers method was also employed for a global/local approach with incompatible FE meshes by introducing an independent displacement field at the interface (three-fields formulation) [15]. Ransom extended this technique for different solution methods, like finite differences, Finite Elements and finite volumes [16]. Note the substantial works of Park and Felippa and their systematic development of hybrid functionals for the analysis of partitioned systems with Lagrange Multipliers $[17,18]$. In these works, the rigid-body modes in the governing equation of floating subdomains are explicitly separated in order to attain the solvability condition. Both static and dynamic problems are addressed. However, these approaches require a degree of freedom compatibility.

As an alternative, Blanco et al. [19] developed the so-called eXtended Variational Formulation (XVF), in which two Lagrange
Multipliers fields are introduced. It allows to couple structural models with different dimensionality involving different types of degrees of freedom. As Lagrange Multipliers can disturb the banded form of the linear system describing the mechanical problem, Kim [20] developed a special interface element, avoiding Lagrange Multipliers. The continuity of the displacements is ensured through the interface element domain with new specific shape functions constructing by moving least square approximations. The numerical integration is a difficult task in this approach.

In commercial FE codes, global/local analysis can be performed. The overall domain is described with a simple or low order kinematical model, and local sub-domains can be modeled by refined descriptions. At the common border, the results of the simple model are used as boundary conditions of the local sub-domain. Calculations are usually carried out in at least two subsequently steps, but iterative methods can also be applied. We can also mention Multiple Point Constraints (MPC) which are available in commercial codes. A kinematics constraint on the degrees of freedom (dofs) can be imposed at the common interface between two models. This technique does not require additional dofs. Nevertheless, it does not rely on an energy principle. Recently, a MPC formulation for eigenfrequency calculation and dynamic contact problems was developed by Hetherington et al. [21].

In the present work, the so-called XVF approach is extended to couple heterogeneous kinematics for the modeling of composite structures within a non-overlapping scheme. The local region of interest is modeled with a refined Sinus approach previously introduced in [22], which has shown very interesting features, while classical simple models can be used for the "global" portion of the structure. A compatibility condition is imposed at the common interface by introducing two Lagrange Multipliers fields that are to be chosen according to the specific kinematics used in the adjacent sub-domains. No additional unknown has to be introduced. The standard MPC approach via penalty method will be addressed for the sake of comparison.

We now outline the remainder of the article. The formulation of the XVF approach is first given. The classical models (namely Euler Bernoulli and Timoshenko) as well as the refined Sinus model are subsequently described. Note that all these models have very different features. The transverse shear stress deduced from these approaches are rather different. Moreover, only the refined model takes into account the transverse normal deformation. We finally focus on the coupling of these different models within the particular XVF framework and on the resulting FEM formulation. Numerical assessments are provided on homogeneous and sandwich beams for both XVF and penalty methods. A preliminary study is devoted to the evaluation of each model without coupling. The influence of the position and the size of the complex zones are discussed. Special attention is also dedicated to the influence of this coupling on the results close to the interface between the domains.

\section{2. eXtended Variational Formulation (XVF)}

Let us consider an elastic structure defined in a domain $\Omega \subset \mathcal{R}^{3}$ bounded by $\Gamma=\Gamma_{D} \cup \Gamma_{N}$ with $\Gamma_{D} \cap \Gamma_{N}=\emptyset$. The structure is submitted to prescribed body forces $\boldsymbol{f}$ on $\Omega$ and surface forces $\boldsymbol{t}$ applied on $\Gamma_{N}$. The displacement $\overline{\boldsymbol{u}}$ is imposed on $\Gamma_{D}$. In the present scope, a beam structure occupying the domain $\Omega=[0, l] \times S$ is addressed, where $S$ is the constant cross-section of the beam, and $l$ is the length. Using the variational formulation, one can state:

Find $\boldsymbol{u} \in \mathcal{U}$ such that:

$$
\int_{\Omega} \boldsymbol{\sigma}(\boldsymbol{u}) \delta \boldsymbol{\epsilon}(\boldsymbol{u}) d \Omega=\int_{\Omega} \boldsymbol{f} \cdot \delta \boldsymbol{u} d \Omega+\int_{\Gamma_{N}} \boldsymbol{t} \cdot \delta \boldsymbol{u} d \partial \Omega \quad \forall \delta \boldsymbol{u} \in \delta \mathcal{U}
$$

where 


$$
\mathcal{U}=\left\{\boldsymbol{u} \in \boldsymbol{H}^{1}(\Omega) ; \boldsymbol{u}_{\mid \Gamma_{D}}=\overline{\boldsymbol{u}}\right\}
$$

is the space of admissible displacements, $\delta \mathcal{U}$ the space obtained from differences between elements of $\mathcal{U}, \boldsymbol{\sigma}$ is the stress tensor and $\boldsymbol{\epsilon}$ is the strain tensor.

In the following, let us denote

$$
\begin{aligned}
& \delta \boldsymbol{\Pi}_{\text {int }}(\boldsymbol{u}, \delta \boldsymbol{u})=\int_{\Omega} \boldsymbol{\sigma}(\boldsymbol{u}) \delta \boldsymbol{\epsilon}(\boldsymbol{u}) d \Omega \\
& \delta \boldsymbol{\Pi}_{\text {ext }}(\delta \boldsymbol{u})=\int_{\Omega} \boldsymbol{f} \cdot \delta \boldsymbol{u} d \Omega+\int_{\Gamma_{N}} \boldsymbol{t} \cdot \delta \boldsymbol{u} d \partial \boldsymbol{\Omega}
\end{aligned}
$$

The domain $\Omega$ is divided by a smooth artificial internal boundary or interface $\Gamma_{a}$ into $\Omega_{c}$ and $\Omega_{s}$ such that $\Omega=\Omega_{s} \cup \Omega_{c}$ and $\Gamma_{a}=\Omega_{s} \cap \Omega_{c}$ (See Fig. 1). So, the new displacement fields ${ }^{s} \boldsymbol{u}$ and ${ }^{c} \boldsymbol{u}$, solution of the problem (1), can be defined in each region. They also have to satisfy the following conditions over the boundary $\Gamma_{a}$ :

${ }^{s} \boldsymbol{u}={ }^{c} \boldsymbol{u} \quad$ in $\boldsymbol{H}^{1 / 2}\left(\Gamma_{a}\right)$

${ }^{s} \boldsymbol{\sigma}^{s} \boldsymbol{n}={ }^{c} \boldsymbol{\sigma}^{c} \boldsymbol{n}$ in $\boldsymbol{H}^{-1 / 2}\left(\Gamma_{a}\right)$

In the work of Blanco et al. [19], XVF was used to couple dimensionally heterogeneous subdomains, which is not the case for the present work. Here, the coupling of different kinematics is our main topics. The subdomain $\Omega_{c}$, called complex zone, is modeled with the refined theory, and $\Omega_{s}$, called simple zone, is modeled with a simpler one. The connection between the subdomains is established via Lagrange Multipliers $\lambda$ at the interface $\Gamma_{a}$.

The variational formulation in Eq. (1) is thus expanded taking into account the terms regarding the interface $\Gamma_{a}$. These additional interface terms establish a vanishing difference in weak sense between the displacements at the interface. A scalar parameter $\gamma$ is also introduced which permits to tune whether simple or complex formulation is used for the connection via Lagrange Multipliers. So, the additional coupling terms, with $\gamma \in[0,1]$ are written as:

$$
\begin{aligned}
& \delta \boldsymbol{\Pi}_{\text {couple }}\left({ }^{s} \boldsymbol{u},{ }^{c} \boldsymbol{u},{ }^{s} \boldsymbol{\lambda},{ }^{c} \boldsymbol{\lambda}, \delta^{s} \boldsymbol{u}, \delta^{c} \boldsymbol{u}, \delta^{s} \boldsymbol{\lambda}, \delta^{c} \boldsymbol{\lambda}\right) \\
& =\gamma \int_{\Gamma_{a}}{ }^{s} \boldsymbol{\lambda} \cdot\left(\delta^{s} \boldsymbol{u}-\delta^{c} \boldsymbol{u}\right) d \Gamma+(1-\gamma) \int_{\Gamma_{a}}{ }^{c} \boldsymbol{\lambda} \cdot\left(\delta^{s} \boldsymbol{u}-\delta^{c} \boldsymbol{u}\right) d \Gamma \\
& \quad+\gamma \int_{\Gamma_{a}} \delta^{s} \boldsymbol{\lambda} \cdot\left({ }^{s} \boldsymbol{u}-{ }^{c} \boldsymbol{u}\right) d \Gamma+(1-\gamma) \int_{\Gamma_{a}} \delta^{c} \boldsymbol{\lambda} \cdot\left({ }^{s} \boldsymbol{u}-{ }^{c} \boldsymbol{u}\right) d \Gamma
\end{aligned}
$$

The notations for this eXtended Variational Formulation are given in Table 1.

Finally, since the subdomains share one common interface, the variational statement of the mechanical problem can be written as follows:

For a given $\gamma \in[0,1]$, find $\left({ }^{s} \boldsymbol{u},{ }^{c} \boldsymbol{u},{ }^{s} \lambda,{ }^{c} \lambda\right) \in \mathcal{U}_{s} \times \mathcal{U}_{c} \times \mathcal{L}_{s} \times \mathcal{L}_{c}$ such that:

$$
\begin{aligned}
\delta \boldsymbol{\Pi}_{\text {int }}\left({ }^{S} \boldsymbol{u}, \delta^{s} \boldsymbol{u}\right)+\delta \boldsymbol{\Pi}_{\text {int }}\left({ }^{c} \boldsymbol{u}, \delta^{c} \boldsymbol{u}\right)= & \delta \boldsymbol{\Pi}_{\text {ext }}\left({ }^{s} \boldsymbol{u}\right)+\delta \boldsymbol{\Pi}_{\text {ext }}\left({ }^{c} \boldsymbol{u}\right) \\
& +\delta \boldsymbol{\Pi}_{\text {couple }}\left({ }^{s} \boldsymbol{u},{ }^{c} \boldsymbol{u},{ }^{s} \boldsymbol{\lambda},{ }^{c} \boldsymbol{\lambda}, \delta^{s} \boldsymbol{u}, \delta^{c} \boldsymbol{u}, \delta^{s} \boldsymbol{\lambda}, \delta^{c} \boldsymbol{\lambda}\right) \\
& \forall\left(\delta^{s} \boldsymbol{u}, \delta^{c} \boldsymbol{u}, \delta^{s} \boldsymbol{\lambda}, \delta^{c} \boldsymbol{\lambda}\right) \in \delta \mathcal{U}_{s} \times \delta \mathcal{U}_{c} \\
& \times \delta \mathcal{L}_{s} \times \delta \mathcal{L}_{c}
\end{aligned}
$$

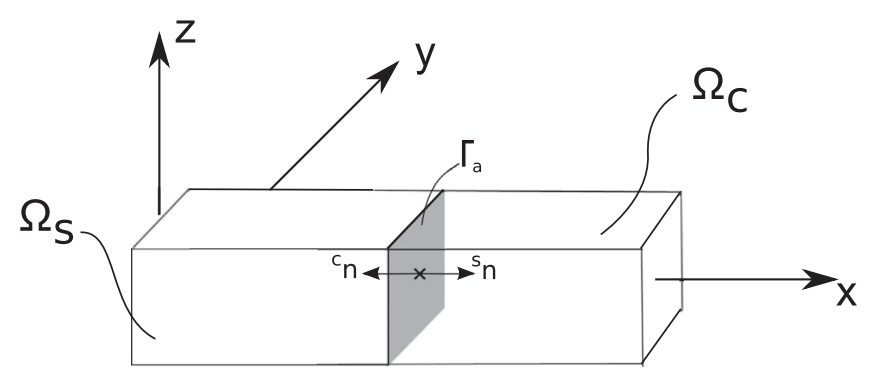

Fig. 1. Complex and simple domains with common interface $\Gamma_{a}$ in a beam structure.
Table 1

Overview of variables in the two subdomains.

\begin{tabular}{lll}
\hline & $\Omega_{s}$ & $\Omega_{c}$ \\
\hline Stress tensor & ${ }^{s} \boldsymbol{\sigma}$ & ${ }^{c} \boldsymbol{\sigma}$ \\
Strain tensor & ${ }^{s} \boldsymbol{\epsilon}$ & ${ }^{c} \boldsymbol{\epsilon}$ \\
Displacement & ${ }^{s} \boldsymbol{u}$ & ${ }^{c} \boldsymbol{u}$ \\
Virtual displacement & $\delta^{s} \boldsymbol{u}$ & $\delta^{c} \boldsymbol{u}$ \\
External traction & ${ }^{s} \boldsymbol{t}$ & ${ }^{c} \boldsymbol{t}$ \\
External force & ${ }^{s} \boldsymbol{f}$ & ${ }^{c} \boldsymbol{f}$ \\
Lagrange Multiplier at interface & ${ }^{s} \boldsymbol{\lambda}$ & ${ }^{c} \boldsymbol{\lambda}$ \\
Virtual Lagrange Multiplier at interface & $\delta^{s} \lambda$ & ${ }^{\delta^{c}} \boldsymbol{\lambda}$ \\
\hline
\end{tabular}

where we have the following definitions:

$\mathcal{U}_{s}=\left\{{ }^{s} \boldsymbol{u} \in \boldsymbol{H}^{1}\left(\Omega_{s}\right) ;{ }^{s} \boldsymbol{u}_{\mid \Gamma_{D}}={ }^{s} \overline{\boldsymbol{u}}\right\}$

$\mathcal{U}_{c}=\left\{{ }^{c} \boldsymbol{u} \in \boldsymbol{H}^{1}\left(\Omega_{c}\right) ;{ }^{c} \boldsymbol{u}_{\mid \Gamma_{D}}={ }^{c} \overline{\boldsymbol{u}}\right\}$

$\mathcal{U}_{s}$ and $\mathcal{U}_{c}$ are the spaces of the admissible displacements. $\mathcal{L}_{s}=\mathcal{L}_{c}=\boldsymbol{H}^{-1 / 2}\left(\Gamma_{a}\right)$.

The Euler-Lagrange equations, derived from Eq. (7), at the interface provide the following relations:

$\left\{\begin{array}{lll}\gamma\left({ }^{s} \boldsymbol{u}-{ }^{c} \boldsymbol{u}\right) & =0 \text { on } \Gamma_{a} \\ (1-\gamma)\left({ }^{s} \boldsymbol{u}-{ }^{c} \boldsymbol{u}\right) & =0 \text { on } \Gamma_{a} \\ { }^{s} \boldsymbol{\sigma}^{s} \boldsymbol{n} & =\gamma^{s} \boldsymbol{\lambda}+(1-\gamma){ }^{c} \boldsymbol{\lambda} & \text { on } \Gamma_{a} \\ { }^{c} \boldsymbol{\sigma}^{c} \boldsymbol{n} & =\gamma^{s} \boldsymbol{\lambda}+(1-\gamma)^{c} \boldsymbol{\lambda} & \text { on } \Gamma_{a}\end{array}\right.$

The Euler-Lagrange equations identify the Lagrange Multipliers as the tractions at the interface and impose the continuity of the displacements in the integral sense. It can be noticed that these tractions correspond to ${ }^{s} \lambda$ for $\gamma=1$ and ${ }^{c} \lambda$ for $\gamma=0$ which characterize the originality of XVF approach.

\section{Description of the kinematics}

Using a unified formulation, the kinematics of the models in the $(x, z)$ coordinate system is assumed to be of the following general form:

$\left\{\begin{array}{l}u_{1}(x, z)=v_{0}(x)-z v_{1}(x)+f(z)\left(v_{1}(x)+\theta(x)\right) \\ u_{3}(x, z)=w_{0}(x)+z w_{1}(x)+z^{2} w_{2}(x)\end{array}\right.$

where $v_{0}, w_{0}$ are the axial and normal displacement while $v_{1}, \theta$ are associated with the rotation of the cross-section. $w_{1}, w_{2}$ are the linear and the quadratic expansion terms for the transverse displacement.

The components of the strains $\epsilon$ can be deduced as:

$\left\{\begin{array}{l}\epsilon_{11}(x, z)=v_{0}^{\prime}(x)-z v_{1}^{\prime}(x)+f(z)\left(v_{1}^{\prime}(x)+\theta^{\prime}(x)\right) \\ \gamma_{13}(x, z)=w_{0}^{\prime}(x)+z w_{1}^{\prime}(x)+z^{2} w_{2}^{\prime}(x)-v_{1}(x)+f(z)^{\prime}\left(v_{1}(x)+\theta(x)\right) \\ \epsilon_{33}(x, z)=w_{1}(x)+2 z w_{2}(x)\end{array}\right.$

Note that the functions $w_{1}$ and $w_{2}$ allow us to take into account the transverse normal strain.

To derive the different kinematical models, we will use the following values for the function $f(z)$, which describe the distribution over the thickness of the transverse shear stress:

$f(z)=0, w_{1}=w_{2}=0, v_{1}=w_{0}^{\prime}$, recovering the Euler-Bernoulli theory

$f(z)=z, w_{1}=w_{2}=0$, recovering the Timoshenko theory

$f(z)=\frac{h}{\pi} \sin \frac{\pi z}{h}, w_{1}=w_{2}=0, \quad$ for the Touratier Sinus theory

$f(z)=\frac{h}{\pi} \sin \frac{\pi z}{h}, \quad$ for the Sin-z2 theory 
where $h$ is the thickness of the beam.

Using Eq. (12) and the Hooke's law $\boldsymbol{\sigma}=\overline{\boldsymbol{C}} \boldsymbol{\epsilon}$, the stresses can be calculated. In the Euler-Bernoulli theory, no transverse shear stress is considered, while the Timoshenko model involves a constant distribution through the thickness. In this case, the classical shear correction factor of $\kappa=\frac{5}{6}$ for rectangular cross-sections is used. Only kinematical theories based on Touratier Sinus have a non constant distribution of the transverse shear stress. Moreover, the transverse normal stress is taken into account only in the Sin-z2 theory.

Hereafter, combinations of the Sin-z2 theory (complex model) with either Euler Bernoulli theory or Timoshenko theory (simple model) will be considered. The Sin-z2 theory will be used in the domains of interest $\left(\Omega_{c}\right)$ where the description of the mechanical quantities must be refined. Therefore, coupling of kinematics with or without transverse shear stress and transverse normal stress will be evaluated in the following.

\section{XVF for kinematical coupling between Sin-z2 and Euler- Bernoulli theory}

In this section, the coupling between incompatible kinematics, namely the Sin-z2 and Euler-Bernoulli theory, are described. The projection between the two spaces are precised deducing the coupling matrices for the problem (Cf. Eq. (7)). Finally, the role of the scalar $\gamma$ is discussed.

Eq. (7) implies the computation of several dual products under the form $\int_{\Gamma_{a}} \delta \lambda \cdot \boldsymbol{u} d \Gamma$. Following Blanco's work, a projection of the complex space into the simple one has to be defined, as for, e.g. $\int_{\Gamma_{a}} \delta^{s} \lambda \cdot{ }^{c} \boldsymbol{u} d \Gamma$. In the sense of the dual product, $\delta^{s} \lambda$ has components which will deliver no work with ${ }^{c} \boldsymbol{u}$.

The displacement field ${ }^{c} \boldsymbol{u}$ is split into two parts as follows:

${ }^{c} \boldsymbol{u}={ }^{c} \boldsymbol{u}_{\|}+{ }^{c} \boldsymbol{u}_{\perp}$

where the orthogonal component with respect to the simple space ${ }^{c} \boldsymbol{u}_{\perp}$ is assumed to verify

$\int_{\Gamma_{a}} \delta^{s} \lambda \cdot{ }^{c} \boldsymbol{u}_{\perp} d \Gamma=0$

The projected displacement field ${ }^{c} \boldsymbol{u}_{\|}$is expressed in the same form as ${ }^{s} \boldsymbol{u}$. Using Eqs. (14) and (15), the relations between the components of ${ }^{c} \boldsymbol{u}_{\|}$and ${ }^{c} \boldsymbol{u}$ can be expressed.

\subsection{Projection of complex into simple kinematics}

The projection is hereafter detailed for the coupling of Euler Bernoulli and Sin-z2 models. From Eqs. (11) and (13), the displacement fields ${ }^{s} \boldsymbol{u}$ and ${ }^{c} \boldsymbol{u}$ of the two models can be expressed as:

- Euler Bernoulli

$$
\left\{\begin{array}{l}
{ }^{s} u_{1}(x, z)={ }^{s} v_{0}(x)-z^{s} w_{0}^{\prime}(x) \\
{ }^{s} u_{3}(x, z)={ }^{s} w_{0}(x)
\end{array}\right.
$$

- Sin-z2

$$
\left\{\begin{array}{l}
{ }^{c} u_{1}(x, z)=v_{0}(x)-z v_{1}(x)+f(z)\left(v_{1}(x)+\theta(x)\right) \\
{ }^{c} u_{3}(x, z)=w_{0}(x)+z w_{1}(x)+z^{2} w_{2}(x)
\end{array}\right.
$$

with

$$
\left\{\begin{array}{l}
{ }^{c} u_{1_{\|}}(x, z)={ }^{c} v_{0_{\|}}(x)-z^{c} w_{0_{\|}}^{\prime}(x) \\
{ }^{c} u_{3_{\|}}(x, z)={ }^{c} w_{0_{\|}}(x)
\end{array}\right.
$$

The Lagrange Multipliers defined on the interface $\Gamma_{a}$ are constructed similarly as the corresponding kinematical field. So, the
Lagrange Multipliers field according to the simple kinematics is expressed as:

$$
\left\{\begin{array}{l}
{ }^{s} \lambda_{1}(x, z)={ }^{s} \lambda_{v_{0}}-z^{s} \lambda_{w_{0}^{\prime}} \\
{ }^{s} \lambda_{3}(x, z)={ }^{s} \lambda_{w_{0}}
\end{array}\right.
$$

Using Eqs. (14) and (15), the applied projection implies:

$$
\begin{aligned}
0= & \int_{\Gamma_{a}} \delta^{s} \lambda \cdot\left({ }^{c} \boldsymbol{u}-{ }^{c} \boldsymbol{u}_{\|}\right) d \Gamma \\
= & \int_{\Gamma} \delta^{s} \lambda_{v_{0}}\left[\left({ }^{c} v_{0}-{ }^{c} v_{0_{\|}}\right)+(f(z)-z)^{c} v_{1}+f(z){ }^{c} \theta-z^{c} w_{0_{\|}^{\prime}}\right] \\
& -z \delta^{s} \lambda_{w_{0}^{\prime}}\left[\left({ }^{c} v_{0}-{ }^{c} v_{0_{\|}}\right)+(f(z)-z)^{c} v_{1}+f(z)^{c} \theta-z^{c} w_{0_{\|}}^{\prime}\right] \\
& +\delta^{s} \lambda_{w_{0}}\left[\left({ }^{c} w_{0}+z^{c} w_{1}+z^{2}{ }^{c} w_{2}-{ }^{c} w_{0_{\|}}\right)\right] d \Gamma
\end{aligned}
$$

Using Eq. (20) and the notations $\int_{\Gamma_{a}} 1 d \Gamma=A$ and $\int_{\Gamma_{a}} z^{2} d \Gamma=I$, the following expressions can be deduced:

$$
\begin{aligned}
& \forall \delta^{s} \lambda_{v_{0}}:{ }^{c} v_{0_{\|}}=\frac{1}{A} \int_{\Gamma_{a}}\left({ }^{c} v_{0}+(f(z)-z)^{c} v_{1}+f(z){ }^{c} \theta-z^{c} w_{0_{\|}}^{\prime}\right) d \Gamma \\
& \forall \delta^{s} \lambda_{w_{0}}:{ }^{c} w_{0_{\|}}=\frac{1}{A} \int_{\Gamma_{a}}\left({ }^{c} w_{0}+z^{c} w_{1}+z^{2}{ }^{c} w_{2}\right) d \Gamma \\
& \forall \delta^{s} \lambda_{w_{0}^{\prime}}:{ }^{c} w_{0_{\|}}^{\prime}=\frac{1}{I} \int_{\Gamma_{a}}\left(z\left({ }^{c} v_{0}-{ }^{c} v_{0_{\|}}\right)+z(f(z)-z)^{c} v_{1}+z f(z){ }^{c} \theta\right) d \Gamma
\end{aligned}
$$

For a symmetric cross-section $\left(\int_{\Gamma_{a}} z d \Gamma=\int_{\Gamma_{a}} f(z) d \Gamma=0\right)$, Eq. (21) gives:

$$
\begin{aligned}
& \forall \delta^{s} \lambda_{v_{0}}:{ }^{c} v_{0_{\|}}={ }^{c} v_{0} \\
& \forall \delta^{s} \lambda_{w_{0}}:{ }^{c} w_{0_{\|}}={ }^{c} w_{0}+\frac{h^{2}}{12}{ }^{c} w_{2} \\
& \forall \delta^{s} \lambda_{w_{0}^{\prime}}:{ }^{c} w_{0_{\|}}^{\prime}=-\frac{24}{\pi^{3}}{ }^{c} \theta-\left(\frac{24}{\pi^{3}}-1\right){ }^{c} v_{1}
\end{aligned}
$$

\subsection{Matrix formulation}

A compact matrix notation for the fields $\boldsymbol{u}$ and $\boldsymbol{\lambda}$ is introduced. The displacement field in Eq. (11) is rewritten using a generalized displacement vector $\mathcal{E}_{u}$ as:

$$
\begin{aligned}
{ }^{i} \boldsymbol{u} & ={ }^{i} \boldsymbol{F}^{i} \mathcal{E}_{u}, i \in\{c, s\} \quad \text { with } \quad{ }^{c} \mathcal{E}_{u}=\left[v_{0} w_{0} v_{1} \theta w_{1} w_{2}\right]^{T}, \quad{ }^{s} \mathcal{E}_{u} \\
& =\left[v_{0} w_{0} w_{0}^{\prime}\right]^{T}
\end{aligned}
$$

and

$$
{ }^{c} \boldsymbol{F}=\left[\begin{array}{cccccc}
1 & 0 & f(z)-z & f(z) & 0 & 0 \\
0 & 1 & 0 & 0 & z & z^{2}
\end{array}\right] \quad{ }^{s} \boldsymbol{F}=\left[\begin{array}{ccc}
1 & 0 & -z \\
0 & 1 & 0
\end{array}\right]
$$

Similarly, the expression of the Lagrange Multipliers are as follows:

${ }^{i} \boldsymbol{\lambda}={ }^{i} \boldsymbol{F}^{i} \mathcal{E}_{\lambda}$ with ${ }^{c} \mathcal{E}_{\lambda}=\left[\lambda_{v_{0}} \lambda_{w_{0}} \lambda_{v_{1}} \lambda_{\theta} \lambda_{w_{1}} \lambda_{w_{2}}\right]^{T}$ and

${ }^{s} \mathcal{E}_{\lambda}=\left[\lambda_{v_{0}} \lambda_{w_{0}} \lambda_{w_{0}^{\prime}}\right]^{T}$

These expressions are particularized for the involved kinematics to deduce the coupling matrices $\boldsymbol{B}_{\times \times}$:

$\int_{\Gamma_{a}} \delta^{s} \lambda \cdot{ }^{s} \boldsymbol{u} d \Gamma=\int_{\Gamma} \delta^{s} \mathcal{E}_{\lambda}^{T s} \boldsymbol{F}^{T}{ }^{s} \boldsymbol{F}^{s} \mathcal{E}_{u} d \Gamma=\delta^{s} \mathcal{E}_{\lambda}^{T} \mathcal{B}_{s s}{ }^{s} \mathcal{E}_{u} \quad$ with

$\mathcal{B}_{s s}=\int_{-\frac{h}{2}}^{\frac{h}{2}}\left(\begin{array}{ccc}1 & 0 & -z \\ 0 & 1 & 0 \\ -z & 0 & z^{2}\end{array}\right) d z$ 
and

$$
\begin{gathered}
\int_{\Gamma_{a}} \delta^{s} \lambda \cdot{ }^{c} \boldsymbol{u} d \Gamma=\int_{\Gamma} \delta^{s} \mathcal{E}_{\lambda}^{T}{ }^{s} \boldsymbol{F}^{T}{ }^{c} \boldsymbol{F}^{c} \mathcal{E}_{u} d \Gamma=\delta^{s} \mathcal{E}_{\lambda}^{T} \mathcal{B}_{s c}{ }^{c} \mathcal{E}_{u} \quad \text { with } \\
\mathcal{B}_{s c}=\int_{-\frac{h}{2}}^{\frac{h}{2}}\left(\begin{array}{ccc}
1 & 0 & -z \\
0 & 1 & 0 \\
-\frac{24 z}{\pi^{3}} & 0 & -\frac{24 z^{2}}{\pi^{3}} \\
\left(1-\frac{24}{\pi^{3}}\right) z & 0 & -\left(1-\frac{24}{\pi^{3}}\right) z^{2} \\
0 & z & 0 \\
0 & z^{2} & 0
\end{array}\right) d z
\end{gathered}
$$

$\mathcal{B}_{c c}$ can also be calculated as:

$$
\begin{aligned}
& \int_{\Gamma_{a}} \delta^{c} \boldsymbol{\lambda} \cdot{ }^{c} \boldsymbol{u} d \Gamma=\delta^{c} \mathcal{E}_{\lambda}^{T} \mathcal{B}_{c c}{ }^{c} \mathcal{E}_{u} \text { with } \\
& \mathcal{B}_{c c}=\int_{-\frac{h}{2}}^{\frac{h}{2}}\left(\begin{array}{cccccc}
1 & 0 & f(z)-z & f(z) & 0 & 0 \\
0 & 1 & 0 & 0 & z & z^{2} \\
f(z)-z & 0 & (f(z)-z)^{2} & (f(z)-z) f(z) & 0 & 0 \\
f(z) & 0 & (f(z)-z) f(z) & (f(z))^{2} & 0 & 0 \\
0 & z & 0 & 0 & z^{2} & z^{3} \\
0 & z^{2} & 0 & 0 & z^{3} & z^{4}
\end{array}\right) d z
\end{aligned}
$$

We also have $\mathcal{B}_{c s}=\mathcal{B}_{s c}^{T}$. So, the matrix expression of Eq. (7) can be written as:

$$
\begin{gathered}
\delta \boldsymbol{\Pi}_{\text {couple }}\left({ }^{s} \mathcal{E}_{u},{ }^{c} \mathcal{E}_{u},{ }^{s} \mathcal{E}_{\lambda},{ }^{c} \mathcal{E}_{\lambda}, \delta^{s} \mathcal{E}_{u}, \delta^{c} \mathcal{E}_{u}, \delta^{s} \mathcal{E}_{\lambda}, \delta^{c} \mathcal{E}_{\lambda}\right)=\left[\begin{array}{c}
\delta^{s} \mathcal{E}_{u} \\
\delta^{c} \mathcal{E}_{u} \\
\delta^{s} \mathcal{E}_{\lambda} \\
\delta^{c} \mathcal{E}_{\lambda}
\end{array}\right]^{T} \\
{\left[\begin{array}{cccc}
0 & 0 & \gamma \mathcal{B}_{s s} & (1-\gamma) \mathcal{B}_{s c} \\
0 & 0 & \gamma \mathcal{B}_{s c}^{T} & (1-\gamma) \mathcal{B}_{c c} \\
\gamma / \mathcal{B}_{s s} & \gamma \mathcal{B}_{s c} & 0 & 0 \\
(1-\gamma) \mathcal{B}_{s c}^{T} & (1-\gamma) \mathcal{B}_{c c} & 0 & 0
\end{array}\right]\left[\begin{array}{c}
{ }^{s} \mathcal{E}_{u} \\
{ }^{c} \mathcal{E}_{u} \\
{ }^{s} \mathcal{E}_{\lambda} \\
{ }^{c} \mathcal{E}_{\lambda}
\end{array}\right]}
\end{gathered}
$$

\subsection{About the choice of $\gamma$}

In Eq. (7), the scalar parameter $\gamma \in[0,1]$ is introduced. In order to evaluate the role of this parameter, Eq. (29) is rewritten considering the two previously involved kinematics (Sin-z2 and EulerBernoulli) and keeping only the terms associated with the variation of the Lagrange Multipliers. The following expression is deduced:

$$
\begin{aligned}
& \delta^{s} \mathcal{E}_{\lambda}^{T} \gamma \mathcal{B}_{s s}{ }^{s} \mathcal{E}_{u}-\delta^{s} \mathcal{E}_{\lambda}^{T} \gamma \mathcal{B}_{s c}{ }^{c} \mathcal{E}_{u}+\delta^{c} \mathcal{E}_{\lambda}^{T}(1-\gamma) \mathcal{B}_{c s}{ }^{s} \mathcal{E}_{u}-\delta^{c} \mathcal{E}_{\lambda}^{T}(1-\gamma) \mathcal{B}_{c c}{ }^{c} \mathcal{E}_{u} \\
& =\gamma \delta^{s} \lambda_{v_{0}}\left[{ }^{s} v_{0}-{ }^{c} v_{0}\right] h+(1-\gamma) \delta^{c} \lambda_{v_{0}}\left[{ }^{s} v_{0}-{ }^{c} v_{0}\right] h \\
& +\gamma \delta^{s} \lambda_{w_{0}}\left[{ }^{s} w_{0}-{ }^{c} w_{0}-\frac{h^{2}}{12}{ }^{c} w_{2}\right] h+(1-\gamma) \delta^{c} \lambda_{w_{0}}\left[{ }^{s} w_{0}-{ }^{c} w_{0}-\frac{h^{2}}{12}{ }^{c} w_{2}\right] h \\
& +\gamma \underbrace{\delta^{s} \lambda_{w_{0}^{\prime}}\left[\frac{h^{3}}{12}{ }^{s} w_{0}^{\prime}-\frac{h^{3}\left(\pi^{3}-24\right)}{12 \pi^{3}} c v_{1}+\frac{2 h^{3}}{\pi^{3}} c \theta\right]}_{I} \\
& +(1-\gamma) \underbrace{\delta^{c} \lambda_{\theta}\left[-\frac{2 h^{3}}{\pi^{3}}{ }^{s} w_{0}^{\prime}-\frac{h^{3}(\pi-4)}{2 \pi^{3}} c v_{1}-\frac{h^{3}}{2 \pi^{2}} c^{c} \theta\right]}_{\text {II }} \\
& +(1-\gamma) \underbrace{\delta^{c} \lambda_{v_{1}}\left[\frac{h^{3}\left(\pi^{3}-24\right)}{12 \pi^{3}} s w_{0}^{\prime}-\frac{h^{3}\left(\pi^{3}+6 \pi-48\right)}{12 \pi^{3}} c v_{1}-\frac{h^{3}(\pi-4)}{2 \pi^{3}} c \theta\right]}_{\text {III }}
\end{aligned}
$$

Applying factorization on the terms I, II and III gives:

$$
\begin{aligned}
& \text { I }: \delta^{s} \lambda_{w_{0}^{\prime}}\left[\left({ }^{s} w_{0}^{\prime}-{ }^{c} v_{1}\right) \frac{h^{3}}{12}+\left({ }^{c} \theta+{ }^{c} v_{1}\right) \frac{2 h^{3}}{\pi^{3}}\right] \\
& \text { II }:-\delta^{c} \lambda_{\theta}\left[\left({ }^{s} w_{0}^{\prime}-{ }^{c} v_{1}\right) \frac{2 h^{3}}{\pi^{3}}+\left({ }^{c} \theta+{ }^{c} v_{1}\right) \frac{h^{3}}{2 \pi^{2}}\right] \\
& \text { III }:-\delta^{c} \lambda_{v_{1}}\left[-\left({ }^{s} w_{0}^{\prime}-{ }^{c} v_{1}\right) \frac{h^{3}}{12}+\left({ }^{s} w_{0}^{\prime}-{ }^{c} \theta-2{ }^{c} v_{1}\right) \frac{2 h^{3}}{\pi^{3}}+\left({ }^{c} \theta+{ }^{c} v_{1}\right) \frac{h^{3}}{2 \pi^{2}}\right]
\end{aligned}
$$

Therefore, we can identify the restrictions satisfying the conditions at the interface. The deduced sets of relations on the displacement components are now discussed. From Eq. (30), two cases can be distinguished:

(i) For $\gamma \in[0,1[$

$$
\begin{aligned}
& { }^{s} v_{0}={ }^{c} v_{0} \\
& { }^{s} w_{0}={ }^{c} w_{0}+\frac{h^{2}}{12}{ }^{c} w_{2} \\
& { }^{c} v_{1}=-{ }^{c} \theta={ }^{s} w_{0}^{\prime}
\end{aligned}
$$

For this case, the conditions show that the rotation of the cross-section and the unknown $v_{1}$ of the complex model have the same value as the derivative of the deflection of the simple model at the interface. It seems to be a strong restriction, the additional variable of the complex model being reduced as only one unique variable of the simple one.

(ii) For $\gamma=1$

$$
\begin{aligned}
& { }^{s} v_{0}={ }^{c} v_{0} \\
& { }^{s} w_{0}={ }^{c} w_{0}+\frac{h^{2}}{12}{ }^{c} w_{2} \\
& { }^{s} w_{0}^{\prime}=\left(1-\frac{24}{\pi^{3}}\right){ }^{c} v_{1}-\frac{24}{\pi^{3}}{ }^{c} \theta
\end{aligned}
$$

We can notice that the first case $(\gamma \in[0,1[)$ implies the most constrained conditions as it involves 4 conditions at the interface compared with the only 3 ones of the second case $(\gamma=1)$. Hence, only two cases will be considered in the numerical examples: (i) $\gamma=0$ and (ii) $\gamma=1$.

\section{Finite Element formulation}

The Finite Element approximations are presented for the generalized displacement and Lagrange Multiplier field (Eqs. (23) and (25)). The description is limited to the elementary stiffness matrix and coupling matrix for brevity reason.

The strain $\epsilon$ is expressed as:

$\boldsymbol{\epsilon}=\boldsymbol{F}_{\epsilon} \mathcal{E}_{\epsilon} \quad$ with $\quad \mathcal{E}_{\epsilon}=\left[v_{0}^{\prime} w_{0}^{\prime} w_{0}^{\prime \prime} v_{1} v_{1}^{\prime} \theta \theta^{\prime} w_{1} w_{1}^{\prime} w_{2} w_{2}^{\prime}\right]^{T}$

Introducing expression (34) into Eq. (3), we can write:

$\delta \boldsymbol{\Pi}_{\text {int }}(\boldsymbol{u})=\int_{l} \delta \mathcal{E}_{\epsilon}^{T} \boldsymbol{k} \mathcal{E}_{\epsilon} d x$

where $\boldsymbol{k}$ is obtained from the following integration over the crosssection:

$\boldsymbol{k}=\int_{S} \boldsymbol{F}_{\epsilon}^{T} \boldsymbol{C} \boldsymbol{F}_{\epsilon} d S$

The expressions of the vector $\boldsymbol{F}_{\boldsymbol{\epsilon}}$ and the matrix $\boldsymbol{k}$ are given in Appendices A and B for the three different models considered in this work. 
The Finite Element approximation can be now introduced in Eqs. (29) and (35). The interpolation of the unknowns is based on quadratic $C^{0}$ continuous Lagrange polynomials (using 3 nodes) for the in-plane displacements or rotations, or by cubic $C^{1}$ continuous Hermite polynomials (using two nodes) for the transverse displacement of the Euler-Bernoulli model. For the functions of the higher-order terms of the transverse displacement, a linear $C^{0}$ continuous interpolation at the 2 inner nodes is used, which allow to condense the dofs at the elementary level. The different FE approximations for the kinematical theories are precised in Fig. 2. An extensive study of the performance and convergence of these beam FEs for the different kinematical models can be found in [23-25]).
Keeping a general notation depending on the used kinematics, matrices $\boldsymbol{N}$ and $\boldsymbol{d} \boldsymbol{N}$ which contain the interpolation functions of the chosen FE approximation and their derivatives can be introduced. So, the generalized displacements can be expressed as

$$
\begin{aligned}
\mathcal{E}_{u} & =\boldsymbol{N} \boldsymbol{q}_{e} \\
\mathcal{E}_{\epsilon} & =\boldsymbol{d} \boldsymbol{N} \boldsymbol{q}_{e}
\end{aligned}
$$

where $\boldsymbol{q}_{e}$ is the dofs vector for one element $e$.

Substituting the expression of $\mathcal{E}_{\epsilon}$ in Eq. (35), the elementary stiffness matrix can be defined by

$$
\delta \boldsymbol{\Pi}_{\text {int }}(\boldsymbol{u})=\delta \mathbf{q}_{e}^{T} \boldsymbol{K}_{e} \boldsymbol{q}_{e} \quad \text { with } \quad \boldsymbol{K}_{e}=\int_{l} \boldsymbol{d} \boldsymbol{N}^{T} \boldsymbol{k} \boldsymbol{d} \mathbf{N} d x
$$

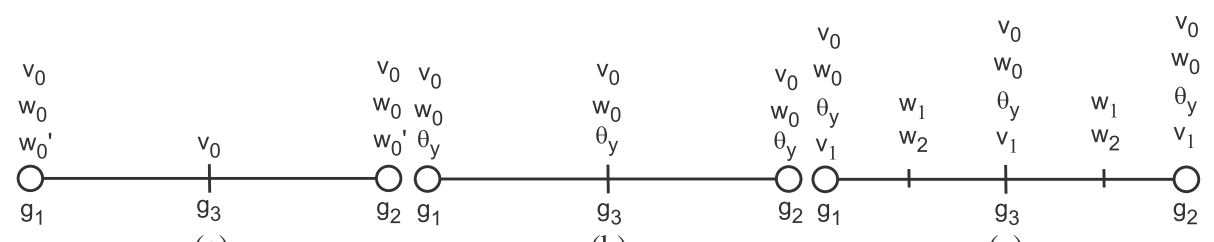

(a)

(b)

(c)

Fig. 2. (a): Euler Bernoulli beam element; (b): Timoshenko beam element; (c): Sin-z2 beam element.

\begin{tabular}{|c|c|c|c|c|c|c|}
\hline & & & $\begin{array}{l}\bar{u}_{3} \\
\left(x=\frac{l}{2}, z=0\right)\end{array}$ & $\begin{array}{l}\bar{\sigma}_{11} \\
\left(x=\frac{l}{2}, z=-\frac{h}{2}\right)\end{array}$ & $\begin{array}{l}\bar{\sigma}_{13} \\
(x=0, z=0)\end{array}$ & $\begin{array}{l}\bar{\sigma}_{13_{\mathrm{EQ}}} \\
(x=0, z=0)\end{array}$ \\
\hline \multirow{4}{*}{$s=5$} & $\begin{array}{l}N_{x}=20 \\
N_{x}=40 \\
N_{x}=60 \\
N_{x}=80\end{array}$ & Euler & $\begin{array}{l}-8583.05 \\
-8583.05 \\
-8583.05 \\
-8583.05 \quad(9.59 \%)\end{array}$ & $\begin{array}{l}0.7141 \\
0.7129 \\
0.7127 \\
0.7126 \quad(0.30 \%)\end{array}$ & $\begin{array}{l}- \\
- \\
- \\
-\end{array}$ & $\begin{array}{l}-0.3750 \\
-0.3750 \\
-0.3750 \\
-0.3750 \quad(1.42 \%)\end{array}$ \\
\hline & $\begin{array}{l}N_{x}=20 \\
N_{x}=40 \\
N_{x}=60 \\
N_{x}=80\end{array}$ & Timo & $\begin{array}{l}-9607.01 \\
-9607.01 \\
-9607.01 \\
-9607.01 \quad(1.19 \%)\end{array}$ & $\begin{array}{l}0.7140 \\
0.7129 \\
0.7127 \\
0.7126 \quad(0.30 \%)\end{array}$ & $\begin{array}{l}-0.2512 \\
-0.2503 \\
-0.2501 \\
-0.2501 \quad(34.25 \%)\end{array}$ & $\begin{array}{l}-0.3662 \\
-0.3690 \\
-0.3696 \\
-0.3698 \quad(2.79 \%)\end{array}$ \\
\hline & $\begin{array}{l}N_{x}=20 \\
N_{x}=40 \\
N_{x}=60 \\
N_{x}=80\end{array}$ & Sin-z2 & $\begin{array}{l}-9454.10 \\
-9454.10 \\
-9454.10 \\
-9454.10(0.42 \%)\end{array}$ & $\begin{array}{l}0.7581 \\
0.7565 \\
0.7562 \\
0.7560 \quad(6.40 \%)\end{array}$ & $\begin{array}{l}-0.3833 \\
-0.3822 \\
-0.3820 \\
-0.3819 \quad(0.39 \%)\end{array}$ & $\begin{array}{l}-0.4118 \\
-0.4120 \\
-0.4121 \\
-0.4121 \quad(8.33 \%)\end{array}$ \\
\hline & & Ansys & -9493.85 & 0.7105 & -0.3804 & - \\
\hline \multirow{4}{*}{$s=10$} & $\begin{array}{l}N_{x}=20 \\
N_{x}=40 \\
N_{x}=60 \\
N_{x}=80\end{array}$ & Euler & $\begin{array}{l}-8582.91 \\
-8582.91 \\
-8582.91 \\
-8582.91 \quad(2.37 \%)\end{array}$ & $\begin{array}{l}0.7141 \\
0.7129 \\
0.7127 \\
0.7126 \quad(0.67 \%)\end{array}$ & $\begin{array}{l}- \\
- \\
- \\
-\end{array}$ & $\begin{array}{l}-0.3750 \\
-0.3750 \\
-0.3750 \\
-0.3750 \quad(1.37 \%)\end{array}$ \\
\hline & $\begin{array}{l}N_{x}=20 \\
N_{x}=40 \\
N_{x}=60 \\
N_{x}=80\end{array}$ & Timo & $\begin{array}{l}-8838.90 \\
-8838.90 \\
-8838.90 \\
-8838.90 \quad(0.54 \%)\end{array}$ & $\begin{array}{l}0.7140 \\
0.7129 \\
0.7127 \\
0.7126 \quad(0.67 \%)\end{array}$ & $\begin{array}{l}-0.2550 \\
-0.2513 \\
-0.2506 \\
-0.2503 \quad(34.17 \%)\end{array}$ & $\begin{array}{l}-0.3735 \\
-0.3746 \\
-0.3748 \\
-0.3749 \quad(1.39 \%)\end{array}$ \\
\hline & $\begin{array}{l}N_{x}=20 \\
N_{x}=40 \\
N_{x}=60 \\
N_{x}=80\end{array}$ & Sin-z2 & $\begin{array}{l}-8783.70 \\
-8783.01 \\
-8783.01 \\
-8783.01 \quad(0.09 \%)\end{array}$ & $\begin{array}{l}0.7272 \\
0.7261 \\
0.7259 \\
0.7258 \quad(1.17 \%)\end{array}$ & $\begin{array}{l}-0.3879 \\
-0.3833 \\
-0.3825 \\
-0.3822 \quad(0.53 \%)\end{array}$ & $\begin{array}{l}-0.4111 \\
-0.4119 \\
-0.4120 \\
-0.4120 \quad(8.36 \%)\end{array}$ \\
\hline & & Ansys & -8791.29 & 0.7174 & -0.3802 & - \\
\hline \multirow{4}{*}{$s=1000$} & $\begin{array}{l}N_{x}=20 \\
N_{x}=40 \\
N_{x}=60 \\
N_{x}=80\end{array}$ & Euler & $\begin{array}{ll}-8582.91 & \\
-8582.91 & \\
-8582.91 & \\
-8582.91 & (0.02 \%)\end{array}$ & $\begin{array}{l}0.7141 \\
0.7129 \\
0.7127 \\
0.7126 \quad(0.01 \%)\end{array}$ & $\begin{array}{l}- \\
- \\
- \\
-\end{array}$ & $\begin{array}{l}-0.3750 \\
-0.3750 \\
-0.3750 \\
-0.3750\end{array}$ \\
\hline & $\begin{array}{l}N_{x}=20 \\
N_{x}=40 \\
N_{x}=60 \\
N_{x}=80\end{array}$ & Timo & $\begin{array}{ll}-8582.91 & \\
-8582.91 & \\
-8582.91 & \\
-8582.91 & (0.02 \%)\end{array}$ & $\begin{array}{l}0.7141 \\
0.7129 \\
0.7127 \\
0.7126 \quad(0.01 \%)\end{array}$ & $\begin{array}{l}-0.2500 \\
-0.2500 \\
-0.2500 \\
-0.2500 \quad(34.02 \%)\end{array}$ & $\begin{array}{l}-0.3750 \\
-0.3750 \\
-0.3750 \\
-0.3750 \quad(1.03 \%)\end{array}$ \\
\hline & $\begin{array}{l}N_{x}=20 \\
N_{x}=40 \\
N_{x}=60 \\
N_{x}=80\end{array}$ & Sin-z2 & $\begin{array}{ll}-8583.00 & \\
-8582.91 & \\
-8582.91 & \\
-8582.91 & (0.02 \%)\end{array}$ & $\begin{array}{l}0.7141 \\
0.7129 \\
0.7127 \\
0.7126(0.01 \%)\end{array}$ & $\begin{array}{l}-0.3627 \\
-0.3627 \\
-0.3627 \\
-0.3627 \quad(4.28 \%)\end{array}$ & $\begin{array}{ll}-0.4121 & \\
-0.4121 & \\
-0.4121 & \\
-0.4121 \quad(8.76 \%)\end{array}$ \\
\hline & & Ansys & -8584.50 & 0.7125 & -0.3789 & - \\
\hline
\end{tabular}

Table 2

Homogeneous Material: assessment of Finite Element approximations. 
In the Finite Element system of the extended Variational Formulation, the stiffness matrices of the domains $\Omega_{s}$ and $\Omega_{c}$ are constructed according to Eq. (38) and are given by:

$\delta \boldsymbol{\Pi}_{\text {int }}\left({ }^{s} \boldsymbol{u}\right)=\delta^{s} \boldsymbol{q} \boldsymbol{K}_{\Omega_{s}}{ }^{s} \boldsymbol{q}$ for $\Omega_{s}$

$\delta \boldsymbol{\Pi}_{\text {int }}\left({ }^{c} \boldsymbol{u}\right)=\delta^{c} \boldsymbol{q} \boldsymbol{K}_{\Omega_{c}}{ }^{c} \boldsymbol{q}$ for $\Omega_{c}$

As far as the additional coupling terms are concerned, a similar procedure is carried out to derive the FE expression of Eq. (29) using the same expression as in Eq. (37) for the Lagrange Multipliers. The discrete expression of $\mathcal{B}_{\times \times}$is written under the form $\boldsymbol{B}_{\times \times}=\boldsymbol{N}^{T} \mathcal{B}_{\times \times} \boldsymbol{N}$.

Finally, after a classical assembling procedure, the global Finite Element system can be deduced for the coupling of two subdomains with one interface:

$$
\left(\begin{array}{cccc}
\boldsymbol{K}_{\Omega_{s}} & 0 & \gamma \boldsymbol{B}_{s s} & (1-\gamma) \boldsymbol{B}_{s c} \\
0 & \boldsymbol{K}_{\Omega_{c}} & \gamma \boldsymbol{B}_{s c}^{T} & (1-\gamma) \boldsymbol{B}_{\boldsymbol{c c}} \\
\gamma \boldsymbol{B}_{s \boldsymbol{s}} & \gamma \boldsymbol{B}_{s c}^{T} & 0 & 0 \\
(1-\gamma) \boldsymbol{B}_{\boldsymbol{s c}} & (1-\gamma) \boldsymbol{B}_{\boldsymbol{c c}} & 0 & 0
\end{array}\right)\left(\begin{array}{c}
{ }^{s} \boldsymbol{q} \\
{ }^{c} \boldsymbol{q} \\
{ }^{s} \boldsymbol{L} \\
{ }^{c} \boldsymbol{L}
\end{array}\right)=\left(\begin{array}{c}
{ }^{s} \boldsymbol{f} \\
{ }^{c} \boldsymbol{f} \\
0 \\
0
\end{array}\right)
$$

where the global Lagrange Multiplier dofs are denoted ${ }^{s} \boldsymbol{L}$ and ${ }^{c} \boldsymbol{L}$.

\section{Results and discussion}

The numerical tests are carried out using the following data:

- Geometry: rectangular beam with $l=10 \mathrm{~mm}$ and slenderness ratio $s=\frac{l}{h}$ of 5,10 and 1000, where $h$ is the thickness of the beam.

- Boundary conditions: simply-supported beam.

- Load: uniform patch load $q_{0}$ on $10 \%$ of the beam length located at the beam center.

- Material properties:

1. Homogeneous Material: $E=1 \mathrm{MPa}$ and $v=0.3$.

2. Sandwich Material:

(a) Face: $E_{11}=131.1 \mathrm{GPa}, E_{22}=E_{33}=6.9 \mathrm{GPa}, G_{12}=3.588 \mathrm{GPa}$, $G_{13}=3.088 \mathrm{GPa}, \quad G_{23}=2.3322 \mathrm{GPa}, \quad v_{12}=v_{13}=0.32$, $v_{23}=0.49$.

(b) Core: $E_{11}=0.2208 \mathrm{MPa}, E_{22}=0.2001 \mathrm{MPa}, E_{33}=2760 \mathrm{MPa}$, $G_{12}=16.56 \mathrm{MPa}, \quad G_{13}=545.1 \mathrm{MPa}, \quad G_{23}=455.4 \mathrm{MPa}$, $v_{12}=0.99, v_{13}=0.00003, v_{23}=0.00003$.

The thicknesses of the layers are: $0.1 h / 0.8 h / 0.1 h$.

- Mesh: half of the beam is meshed, $N_{x}=20,40,60,80\left(N_{x}\right.$ is the number of FEs).

Table 3

Sandwich Material: assessment of Finite Element approximations.

\begin{tabular}{|c|c|c|c|c|c|c|}
\hline & & & $\begin{array}{l}\bar{u}_{3} \\
\left(x=\frac{l}{2}, z=0\right)\end{array}$ & $\begin{array}{l}\bar{\sigma}_{11} \\
\left(x=\frac{l}{2}, z=-\frac{h}{2}\right)\end{array}$ & $\begin{array}{l}\bar{\sigma}_{13} \\
(x=0, z=0)\end{array}$ & $\begin{array}{l}\bar{\sigma}_{13_{\mathrm{EQ}}} \\
(x=0, z=0)\end{array}$ \\
\hline \multirow{4}{*}{$S=5$} & $\begin{array}{l}N_{x}=20 \\
N_{x}=40 \\
N_{x}=60 \\
N_{x}=80\end{array}$ & Euler & $\begin{array}{l}-0.26832 \\
-0.26832 \\
-0.26832 \\
-0.26832 \quad(80.95 \%)\end{array}$ & $\begin{array}{l}0.29265 \\
0.29217 \\
0.29208 \\
0.29205 \quad(37.29 \%)\end{array}$ & $\begin{array}{l}- \\
- \\
- \\
-\end{array}$ & $\begin{array}{l}-0.05533 \\
-0.05533 \\
-0.05533 \\
-0.05533(0.02 \%)\end{array}$ \\
\hline & $\begin{array}{l}N_{x}=20 \\
N_{x}=40 \\
N_{x}=60 \\
N_{x}=80\end{array}$ & Timo & $\begin{array}{l}-1.01485 \\
-1.01485 \\
-1.01485 \\
-1.01485 \quad(27.95 \%)\end{array}$ & $\begin{array}{l}0.29265 \\
0.29217 \\
0.29208 \\
0.29205 \quad(37.29 \%)\end{array}$ & $\begin{array}{l}-0.02587 \\
-0.02587 \\
-0.02587 \\
-0.02587 \quad(53.24 \%)\end{array}$ & $\begin{array}{l}-0.05533 \\
-0.05533 \\
-0.05533 \\
-0.05533(0.02 \%)\end{array}$ \\
\hline & $\begin{array}{l}N_{x}=20 \\
N_{x}=40 \\
N_{x}=60 \\
N_{x}=80\end{array}$ & Sin-z2 & $\begin{array}{l}-1.32690 \\
-1.32690 \\
-1.32690 \\
-1.32690 \quad(5.80 \%)\end{array}$ & $\begin{array}{l}0.53668 \\
0.53388 \\
0.53336 \\
0.53316(14.48 \%)\end{array}$ & $\begin{array}{l}-0.06613 \\
-0.06612 \\
-0.06612 \\
-0.06612 \quad(19.52 \%)\end{array}$ & $\begin{array}{l}-0.05562 \\
-0.05563 \\
-0.05563 \\
-0.05563(0.56 \%)\end{array}$ \\
\hline & & Ansys & -1.40859 & 0.46572 & -0.05532 & - \\
\hline \multirow{4}{*}{$s=10$} & $\begin{array}{l}N_{x}=20 \\
N_{x}=40 \\
N_{x}=60 \\
N_{x}=80\end{array}$ & Euler & $\begin{array}{l}-0.26831 \\
-0.26831 \\
-0.26831 \\
-0.26831 \quad(52.31 \%)\end{array}$ & $\begin{array}{ll}0.29265 & \\
0.29217 & \\
0.29208 & \\
0.29205 \quad(21.40 \%)\end{array}$ & $\begin{array}{l}- \\
- \\
-\end{array}$ & $\begin{array}{l}-0.05533 \\
-0.05533 \\
-0.05533 \\
-0.05533(0 \%)\end{array}$ \\
\hline & $\begin{array}{l}N_{x}=20 \\
N_{x}=40 \\
N_{x}=60 \\
N_{x}=80\end{array}$ & Timo & $\begin{array}{l}-0.45494 \\
-0.45494 \\
-0.45494 \\
-0.45494 \quad(19.13 \%)\end{array}$ & $\begin{array}{l}0.29265 \\
0.29217 \\
0.29208 \\
0.29205 \quad(21.40 \%)\end{array}$ & $\begin{array}{l}-0.02589 \\
-0.02587 \\
-0.02587 \\
-0.02587 \quad(53.24 \%)\end{array}$ & $\begin{array}{l}-0.05532 \\
-0.05533 \\
-0.05533 \\
-0.05533(0 \%)\end{array}$ \\
\hline & $\begin{array}{l}N_{x}=20 \\
N_{x}=40 \\
N_{x}=60 \\
N_{x}=80\end{array}$ & Sin-z2 & $\begin{array}{l}-0.53710 \\
-0.53709 \\
-0.53709 \\
-0.53709(4.53 \%)\end{array}$ & $\begin{array}{ll}0.37522 & \\
0.37388 & \\
0.37365 & \\
0.37357 & (0.54 \%)\end{array}$ & $\begin{array}{l}-0.06615 \\
-0.06613 \\
-0.06613 \\
-0.06613 \quad(19.52 \%)\end{array}$ & $\begin{array}{ll}-0.05562 & \\
-0.05563 & \\
-0.05563 & \\
-0.05563 & (0.54 \%)\end{array}$ \\
\hline & & Ansys & -0.56258 & 0.37157 & -0.05533 & - \\
\hline \multirow{4}{*}{$s=1000$} & $\begin{array}{l}N_{x}=20 \\
N_{x}=40 \\
N_{x}=60 \\
N_{x}=80\end{array}$ & Euler & $\begin{array}{ll}-0.26831 & \\
-0.26831 & \\
-0.26831 & \\
-0.26831 \quad(0.02 \%)\end{array}$ & $\begin{array}{l}0.29265 \\
0.29217 \\
0.29208 \\
0.29205 \quad(0.01 \%)\end{array}$ & $\begin{array}{l}- \\
- \\
- \\
-\end{array}$ & $\begin{array}{l}-0.05533 \\
-0.05533 \\
-0.05533 \\
-0.05533(0 \%)\end{array}$ \\
\hline & $\begin{array}{l}N_{x}=20 \\
N_{x}=40 \\
N_{x}=60 \\
N_{x}=80\end{array}$ & Timo & $\begin{array}{ll}-0.26833 & \\
-0.26833 & \\
-0.26833 & \\
-0.26833 & (0.01 \%)\end{array}$ & $\begin{array}{ll}0.29265 & \\
0.29217 & \\
0.29208 & \\
0.29205 & (0.01 \%)\end{array}$ & $\begin{array}{l}-0.02587 \\
-0.02587 \\
-0.02587 \\
-0.02587 \quad(53.24 \%)\end{array}$ & $\begin{array}{l}-0.05533 \\
-0.05533 \\
-0.05533 \\
-0.05533(0 \%)\end{array}$ \\
\hline & $\begin{array}{l}N_{x}=20 \\
N_{x}=40 \\
N_{x}=60 \\
N_{x}=80\end{array}$ & Sin-z2 & $\begin{array}{ll}-0.26833 & \\
-0.26833 & \\
-0.26833 & \\
-0.26833 & (0.01 \%)\end{array}$ & $\begin{array}{l}0.29266 \\
0.29218 \\
0.29209 \\
0.29206(0.01 \%)\end{array}$ & $\begin{array}{l}-0.06761 \\
-0.06761 \\
-0.06761 \\
-0.06761 \quad(22.19 \%)\end{array}$ & $\begin{array}{l}-0.05563 \\
-0.05563 \\
-0.05563 \\
-0.05563(0.54 \%)\end{array}$ \\
\hline & & Ansys & -0.26836 & 0.29203 & -0.05533 & - \\
\hline
\end{tabular}


- Results are normalized using: $\bar{u}_{3}=100 u_{3}(l / 2,0) \frac{Y_{0}}{h^{4} q_{0}}$, $\bar{\sigma}_{11}=\frac{\sigma_{11}(l / 2,-h / 2)}{s^{2} q_{0}}, \bar{\sigma}_{13}=\frac{\sigma_{13}(0,0)}{s q_{0}}$, with $Y_{0}=E_{33}^{\text {Face }} \cdot \bar{\sigma}_{13_{\mathrm{EQ}}}$ will identify the transverse shear stress computed from the integration of the equilibrium equations.

\subsection{Assessment of models}

In this section, only one model will be used for the whole structure (denoted monomodel) avoiding the kinematical coupling effect of the XVF. It allows us to highlight the effect of each model on the results. The different models (Euler-Bernoulli denoted Euler, Timoshenko denoted Timo, Sin-z2) are assessed on both a homogeneous and a sandwich beam for various slenderness ratios and for different meshes. The results are summarized in Tables 2 and 3. They are compared with FE results issued from a 2D analysis using PLANE82 elements, performed with the commercial code ANSYS with converged meshes. These are reported in Table 4. These tables show that a mesh of $N_{x}=20$ elements provides converged results for thick to very thin beams with $s=5,10,1000$. For the very thin case, the results are rather similar for the three models except for the transverse shear stress. The Sin-z2 yield more accurate results. For very thick and moderately thick structures, the accuracy of the deflection and the transverse shear stress is also improved by the use of the Sin-z2 model.

For further comparison, Figs. 3 and 4 show the deflection of the beam for the two materials. The main differences occur for the sandwich structure. Euler-Bernoulli model yields poor results. The maximum deflection of the Timoshenko model is 20\% greater than the reference solution, whereas the Sin-z2 results are satisfactory with error rate less than $4.5 \%$. The distribution of the transverse shear stress along the thickness is also represented in Figs. 5 and 6. As expected, the results computed from the constitutive relation for the Sin-z2 model are closer to the reference solution than those obtained from the simple models. Using the

\section{Table 4}

ANSYS meshes with PLANE82 elements for homogeneous and sandwich beams.

\begin{tabular}{lllll}
\hline & Homogeneous & \multicolumn{3}{c}{ Sandwich } \\
\hline $\mathrm{s}$ & $N_{x}$ & $N_{z}$ & $N_{x}$ & $N_{\text {face }} / N_{\text {core }} / N_{\text {face }}$ \\
5 & 80 & 20 & 80 & $12 / 48 / 12$ \\
10 & 80 & 10 & 80 & $6 / 24 / 6$ \\
1000 & 720 & 6 & 720 & $2 / 4 / 2$ \\
\hline
\end{tabular}

$N_{z}$ is the number of elements across the thickness.

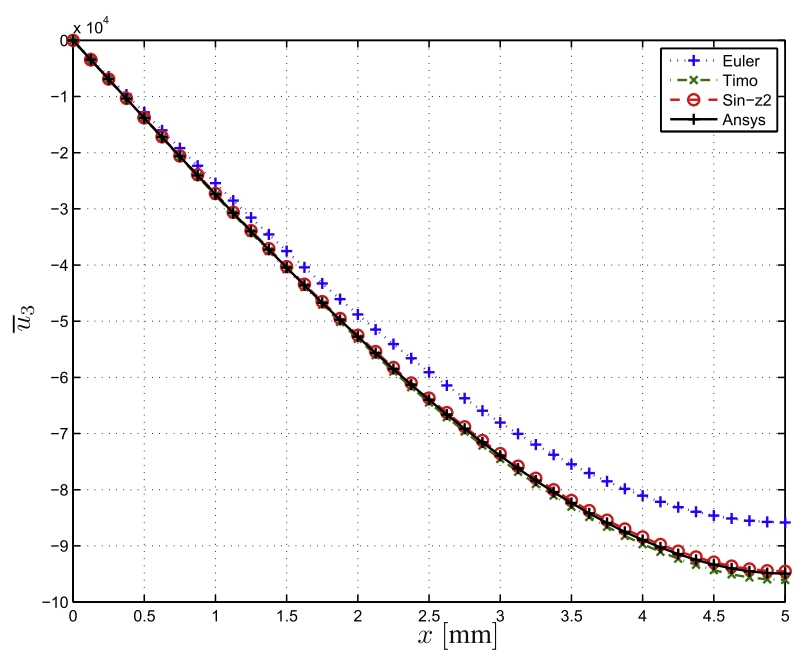

Fig. 3. Homogeneous Material: deflection of the different Kinematics; $s=5$. integration of the equilibrium equations, all the results are in rather good agreement with the ANSYS solutions.

In the following, the simple kinematical model will be either Euler-Bernoulli or Timoshenko kinematics; the complex model will be the Sin-z2 kinematics. While Tables 2 and 3 show that a $N_{x}=20$ mesh is converged, $N_{x}=40$ will be used in the following in order to ensure that all the observed effects are due to the coupling method only.

\subsection{Homogeneous Material structures}

\subsubsection{One interface}

For a preliminary assessment, only one interface is considered. So, two configurations are possible, as illustrated in Fig. 7: the complex zone, namely the region associated with the complex model, can include either the support, or the load. Both configurations are tested with the three coupling approaches: XVF with $\gamma=0, \gamma=1$ and the penalty method.

In Fig. 8, both configurations are tested with the interface located at $\bar{x}=\frac{l}{4}$. Euler-Bernoulli kinematics is used as simple model and Sin-z2 kinematics as complex model. This figure represents the displacement over the length for $\gamma=1$. The same tendencies are obtained for $\gamma=0$ and penalty method. The displacements

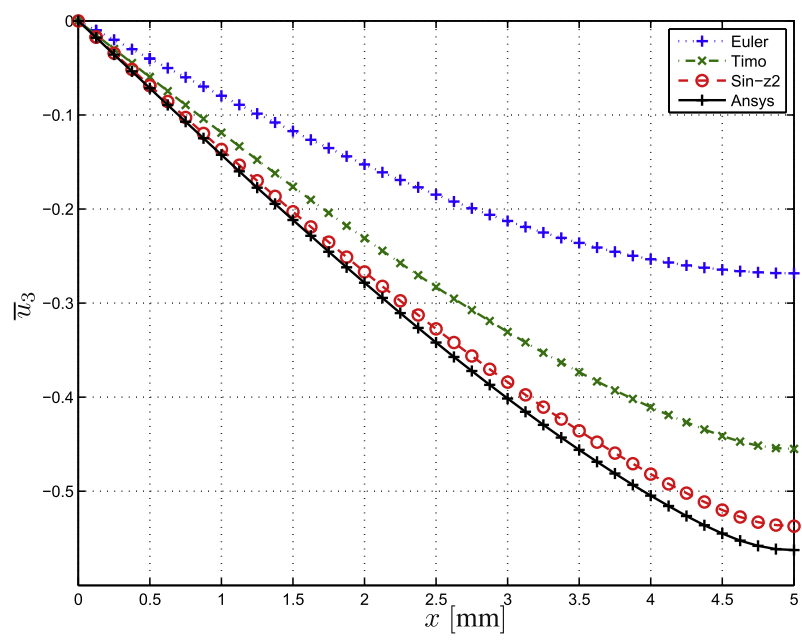

Fig. 4. Sandwich Material: deflection of the different Kinematics; $s=10$.
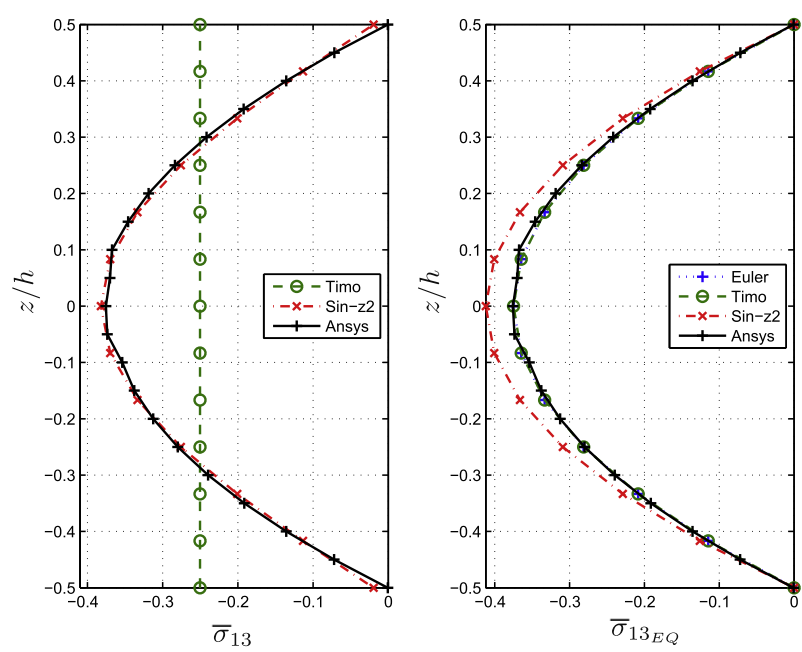

Fig. 5. Homogeneous Material: $\bar{\sigma}_{13}$ through thickness for different kinematics; $s=5$. 

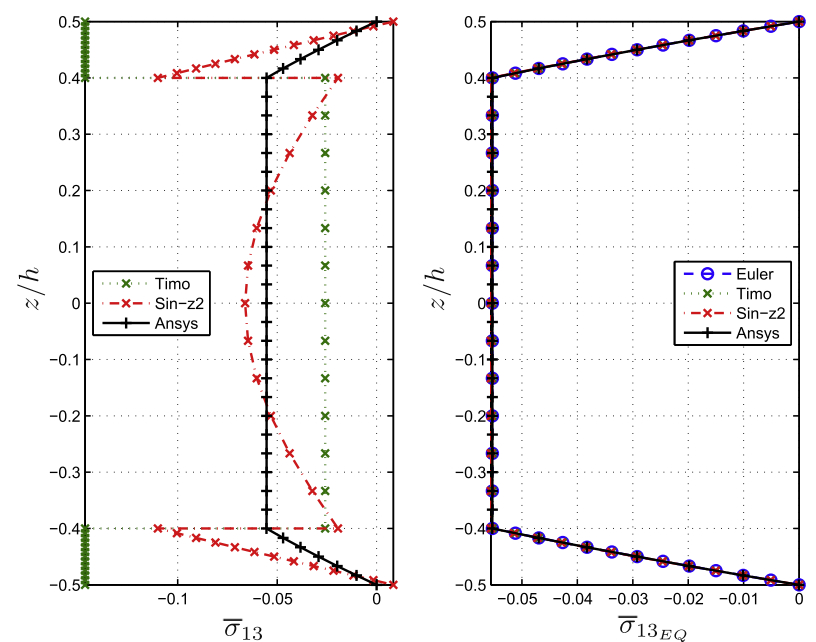

Fig. 6. Sandwich Material: $\bar{\sigma}_{13}$ through thickness for different kinematics; $s=10$.

of the monomodel are also drawn to measure the influence of the coupling. This figure shows that the tendency of the involved model in each subdomain is kept. Moreover, the maximum deflection is influenced by the coupling. The maximum values are in between the results of the two monomodels.

In Table 5, the three computational approaches are assessed for the thick structure which is the most severe case. The deflection and only the maximum stresses computed by the complex model are given. They are compared with the monomodel results. First of all, it can be noticed that the XVF approach with $\gamma=0$ and the penalty method gives always the same results. Furthermore, only the value of the maximal deflection changes with the involved technique of coupling. Since the displacement is a global quantity, it depends on both the choice of the simple model (Euler-Bernoulli or Timoshenko) and the domain modeled by the complex kinematics. On the contrary, the stresses computed in the complex domain

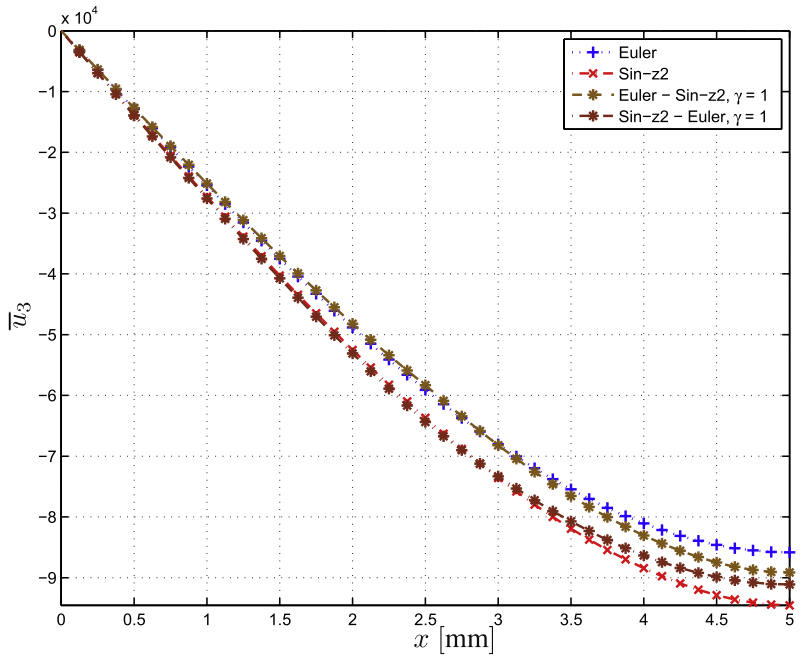

Fig. 8. Homogeneous Material: deflection for $\gamma=1 ; \Gamma_{a}$ at $\bar{x}=2.5 ; s=5$.

is independent of the coupling of kinematics and of the choice of the simple model. The accuracy of its value corresponds only to these of the complex kinematics. This feature is very attractive in the framework of the design of composite structures in which the computation of the stresses in the region of interest is of major importance. Moreover, the number of dofs for the monomodels and the coupling of different kinematics are also compared in Table 5. For this simple example, the decreasing of the computational cost is up to about $60 \%$.

In this first test, the interface position has been kept fixed. In order to assess the sensitivity of the size of the complex zone on the results, the interface will now be located at different positions along beam's axis. The size of the complex subdomain is chosen proportional to the thickness $h$ of the beam and is denoted $\alpha h$, with $\alpha \in\{0.5,1,1.5,2\}$, as illustrated in Fig. 9. Since $s=\frac{l}{h}=5$, the size of the domain is $\alpha \frac{l}{5}$. The results are summarized in Tables 6-8.
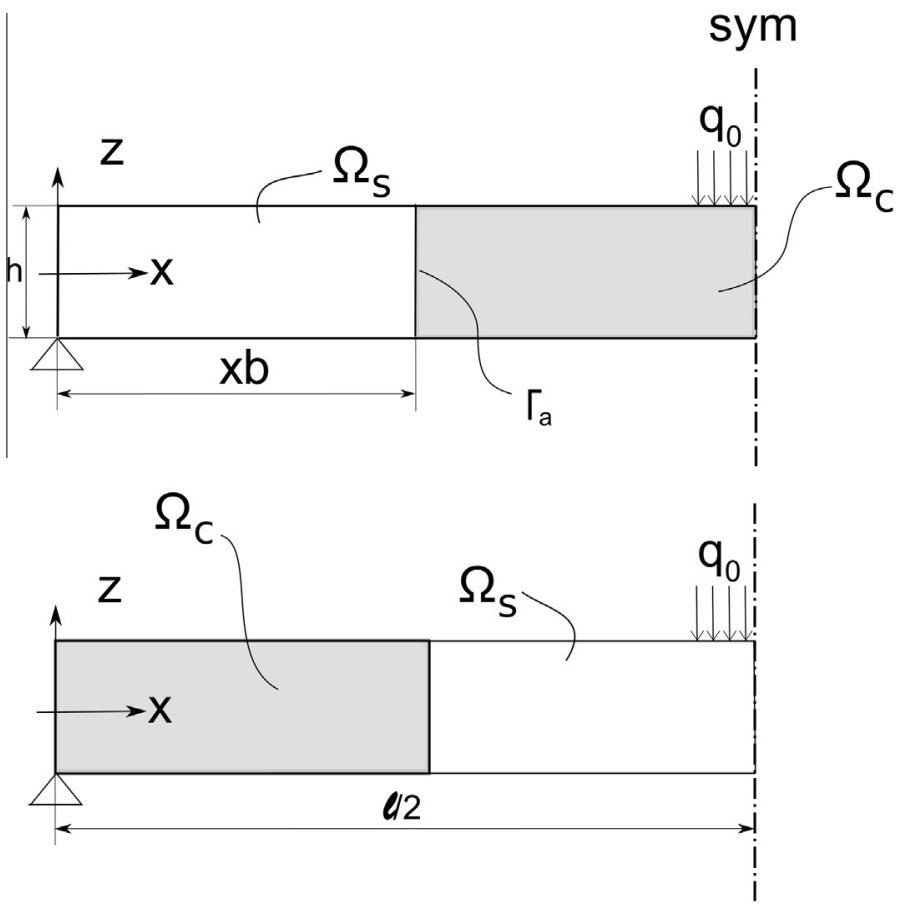

Fig. 7. One interface: configuration of simple and complex domains. 
Table 5

Homogeneous Material: values of $\bar{u}_{3}, \bar{\sigma}_{11}$ and $\bar{\sigma}_{13}$ for $\Gamma_{a}$ at $\bar{x}=2.5$ and for monomodels, $s=5$.

\begin{tabular}{|c|c|c|c|c|c|}
\hline \multirow[b]{2}{*}{ Type } & \multicolumn{2}{|c|}{ Euler-Sin-z2 } & \multicolumn{2}{|c|}{ Sin-z2-Euler } & \multirow[t]{2}{*}{$\mathrm{Nb}$ of dofs } \\
\hline & $\bar{u}_{3}$ & $\bar{\sigma}_{11}$ & $\bar{u}_{3}$ & $\bar{\sigma}_{13}$ & \\
\hline \multirow{4}{*}{$\begin{array}{l}\text { Penalty } \\
\gamma=0 \\
\gamma=1\end{array}$} & -8904.31 & 0.7564 & -9111.31 & -0.3821 & \multirow{5}{*}{199} \\
\hline & -8904.31 & 0.7564 & -9111.31 & -0.3821 & \\
\hline & -8915.35 & 0.7565 & -9121.80 & -0.3822 & \\
\hline & \multicolumn{2}{|c|}{ Timo-Sin-z2 } & \multicolumn{2}{|c|}{ Sin-z2-Timo } & \\
\hline Type & $\bar{u}_{3}$ & $\bar{\sigma}_{11}$ & $\bar{u}_{3}$ & $\bar{\sigma}_{13}$ & \\
\hline Penalty & -9443.62 & 0.7564 & -9595.97 & -0.3821 & \\
\hline$\gamma=0$ & -9443.62 & 0.7564 & -9595.97 & -0.3821 & 271 \\
\hline \multirow[t]{2}{*}{$\gamma=1$} & -9454.10 & 0.7565 & -9607.01 & -0.3822 & \\
\hline & \multicolumn{4}{|c|}{ Monomodel } & \\
\hline Type & $\bar{u}_{3}$ & $\bar{\sigma}_{11}$ & & $\bar{\sigma}_{13}$ & \\
\hline Euler Bernoulli & -8583.05 & 0.7129 & & - & 163 \\
\hline Timoshenko & -9607.01 & 0.7129 & & -0.2503 & 243 \\
\hline Sin-z2 & -9454.10 & 0.7565 & & -0.3822 & 484 \\
\hline
\end{tabular}

We notice that the size of the complex zone has small influence on the deflection and the in-plane stress regardless of the technique of coupling. The transverse shear stress seems to be a little more sensitive for the penalty and XVF approach with $\gamma=0$, see Fig. 10.

\subsubsection{Two interfaces}

As the general interest is to obtain reliable results at the support and under the load simultaneously, complex models will be applied hereafter at both ends. Hence, two interfaces are needed as shown in Fig. 11. The $N_{x}=40$ mesh is divided into 20 elements in the central part of the beam associated with the simple model and 10 elements on each end of the beam for the complex model. For a regular mesh, the two interfaces are located at $\bar{x}_{1}=\frac{l}{8}$ and $\bar{x}_{2}=\frac{3 l}{8}$. Here, the aim is to study the impact of the local refinements on the results of the whole beam structure. Therefore results obtained by Ansys are given for comparison.

Fig. 12 shows the distribution of $\bar{\sigma}_{13}$ at the beam mid fiber, $z=0$, along the beam's axis for monomodels and coupling models. The penalty technique is not presented as it provides the same results as the XVF approach with $\gamma=0$. For $\gamma=0$, the coupling of the models does not influence the results far from the interface, that is to say, the results at a given point correspond to those of the local model. For $\gamma=1$, the same comment can be made even near the interface, the transition between one model to another one being very rapid. So, the quality of the stress obtained by the refined model in the region of interest is affected by neither the introduction of coupling of multi-models nor the choice of the simple model.

\subsection{Layered structures}

In this section, layered structures are addressed. First, we show that is needed to introduce a new coupling operator which takes into account the material characteristics of the layers by considering a highly anisotropic sandwich beam. Then, it is applied to the sandwich structure previously described in Section 6 for further assessment.

\subsubsection{The coupling operator}

In this section, two different dual products are used: one using the original form as given in Eq. (7) and one modified by including also the stiffness coefficient $C_{11}$. This latter is described as follows:

The four dual products of the Eqs. (26)-(28) for the layered structures are integrated over all the layers, denoted $\left\{1, \ldots, N_{L}\right\}$ :

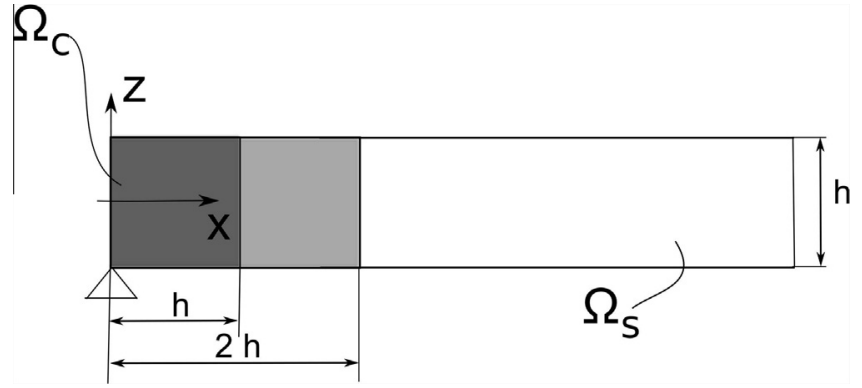

Fig. 9. Size of the complex zone (several times the beam height).

Table 6

Homogeneous Material: values of $\bar{u}_{3}$; Timo-Sin-z2 or Sin-z2-Timo, s $=5$.

\begin{tabular}{lllll}
\hline \multicolumn{5}{l}{ Complex zone at support } \\
\hline Type & $\bar{u}_{3}$ & & & \\
\cline { 2 - 5 } & $0.5 \cdot h$ & $1 \cdot h$ & $1.5 \cdot h$ & $2 \cdot h$ \\
\hline Penalty & -9596.52 & -9596.52 & -9595.97 & -9595.97 \\
$\gamma=0$ & -9596.52 & -9596.52 & -9595.97 & -9595.97 \\
$\gamma=1$ & -9607.01 & -9607.01 & -9607.01 & -9606.46 \\
Complex zone under load & & & \\
\hline Type & $0.5 \cdot h$ & $1 \cdot h$ & $1.5 \cdot h$ & $2 \cdot h$ \\
\hline Penalty & -9445.80 & -9443.62 & -9443.62 & -9443.62 \\
$\gamma=0$ & -9445.80 & -9443.62 & -9443.62 & -9443.62 \\
$\gamma=1$ & -9454.66 & -9454.10 & -9454.10 & -9454.10 \\
\hline
\end{tabular}

Table 7

Homogeneous Material: values of $\bar{\sigma}_{11}$; Timo-Sin-z2, $s=5$.

\begin{tabular}{|c|c|c|c|c|}
\hline \multicolumn{5}{|c|}{ Complex zone under load } \\
\hline Type & $0.5 \cdot h$ & $1 \cdot h$ & $1.5 \cdot h$ & $2 \cdot h$ \\
\hline Penalty & 0.7502 & 0.7562 & 0.7565 & 0.7565 \\
\hline$\gamma=0$ & 0.7502 & 0.7562 & 0.7565 & 0.7565 \\
\hline$\gamma=1$ & 0.7561 & 0.7565 & 0.7565 & 0.7565 \\
\hline
\end{tabular}

Table 8

Homogeneous Material: values of $\bar{\sigma}_{13}$; Sin-z2-Timo, $s=5$

\begin{tabular}{lllll}
\hline \multicolumn{5}{l}{ Refined at support } \\
\cline { 2 - 5 } Type & $\bar{\sigma}_{13}$ & & & \\
\cline { 2 - 5 } & $0.5 \cdot h$ & $1 \cdot h$ & $1.5 \cdot h$ & $2 \cdot h$ \\
\hline Penalty & -0.3731 & -0.3819 & -0.3822 & -0.3822 \\
$\gamma=0$ & -0.3731 & -0.3819 & -0.3822 & -0.3822 \\
$\gamma=1$ & -0.3822 & -0.3822 & -0.3822 & -0.3822
\end{tabular}

$\delta^{i} \mathcal{E}_{\lambda}^{T} \boldsymbol{B}_{i j_{\text {mod }}}{ }^{j} \mathcal{E}_{u} \quad$ with $\quad \boldsymbol{B}_{i j_{\text {mod }}}=\sum_{k=1}^{N_{L}} C_{11}^{(k)} \int_{h_{k-1}}^{h_{k}}{ }^{i} \boldsymbol{F}^{T j} \boldsymbol{F} d z$

The sandwich test described in Section 6 is considered with two interfaces, but the material properties is chosen such that $C_{11_{\text {face }}}=1000 \cdot C_{11_{\text {core }}}$ so as to highlight the interest of the new coupling operator. In Fig. 13, the distribution of the transverse shear stress $\bar{\sigma}_{13}$ through the beam length is given. For $\gamma=1$ without the modified dual product, the distribution of the stress has a severe peak close to the interface, while the same abrupt change from one model to another one as in the homogeneous material case can be observed with the modified duality product. In the Euler Lagrange Eq. (10), the Lagrange Multipliers were identified as stresses. For the proper establishment of the Multipliers not only the geometric informations are needed but also about the materials 


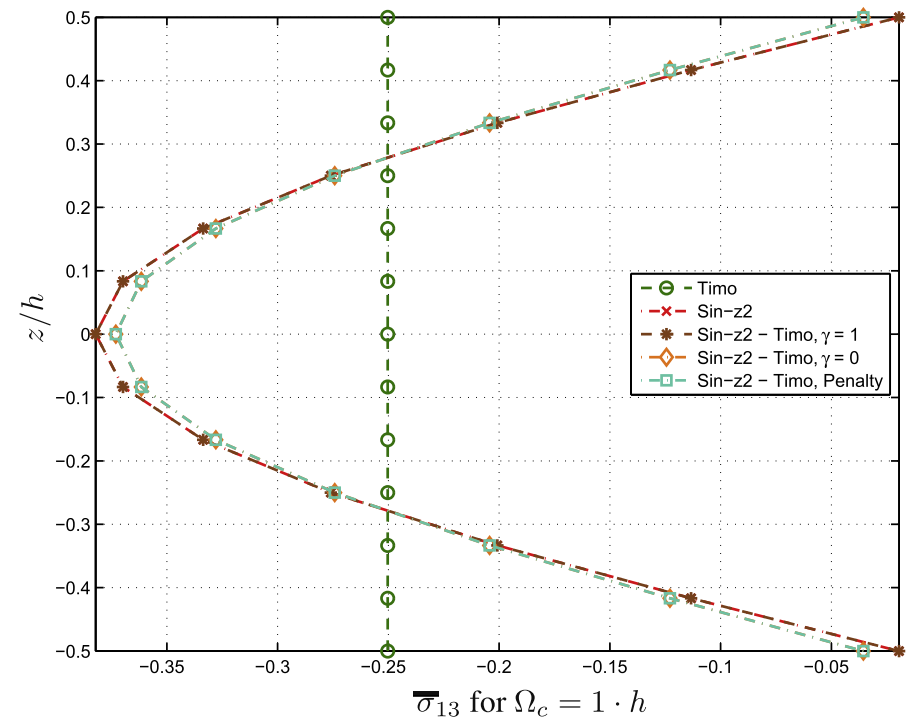

(a)

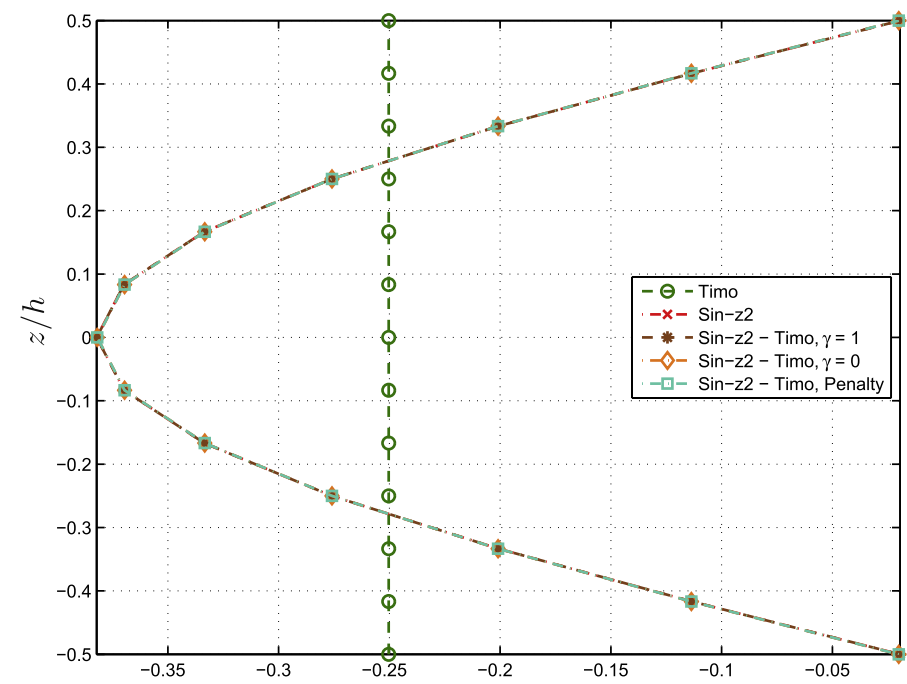

(b)

$\bar{\sigma}_{13}$ for $\Omega_{c}=2 \cdot h$

Fig. 10. Homogeneous Material: (a): $\bar{\sigma}_{13}$ for $\Omega_{c}=1 \cdot h$; (b): $\bar{\sigma}_{13}$ for $\Omega_{c}=2 \cdot h$; refined model at support; $s=5$.

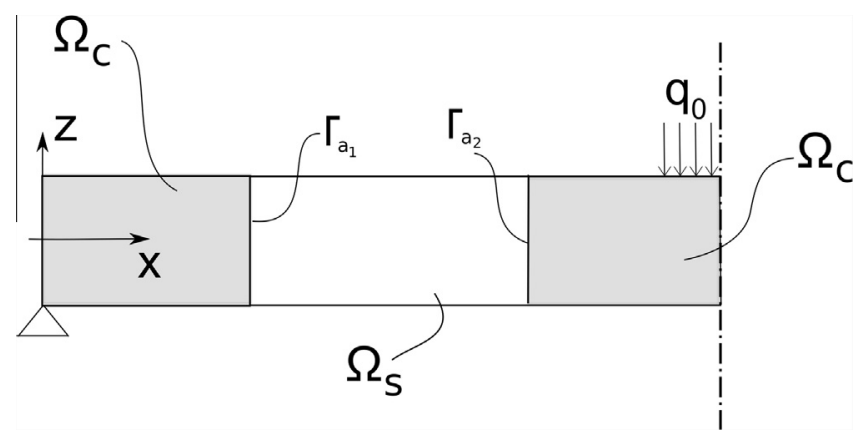

Fig. 11. Position of complex zones in beam model.

stiffness. As a consequence the origin of the peak is a ill-conditioned problem of the original dual product formulation. The values of the Lagrange Multipliers of the original dual product are excessive and, as shown, do not fulfill their role as to satisfactorily minimize the difference in the two displacement fields. Here, the mechanical description of the layered structure is given by an equivalent single layer formulation. It is not visible for homogenous materials, as the stiffness coefficient $C_{11}$ is only a constant factor, regardless of the kinematical model chosen. In contrast, for layered structures, different layers contribute with their geometrical share of the compound as well as with their stiffness. The modification of the formulation is made according to a proposition of $\mathrm{Hu}[26,7]$ for the Arlequin Method.

\subsubsection{Sandwich beam}

The sandwich structure having two thin rigid outer faces and a soft core, as shown in Fig. 14 and given in Section 6, is addressed. Two interfaces are considered as shown in Fig. 11, and the new coupling dual product given in Eq. (41) is used.

The distributions of $\bar{\sigma}_{11}$ and $\bar{\sigma}_{13}$ along the beam length are represented in Figs. 15 and 16 for the monomodels, the Sin-z2Euler-Sin-z2 and Sin-z2-Timo-Sin-z2 couplings. For the in-plane stress, the choice of the simple model has no influence, so only 

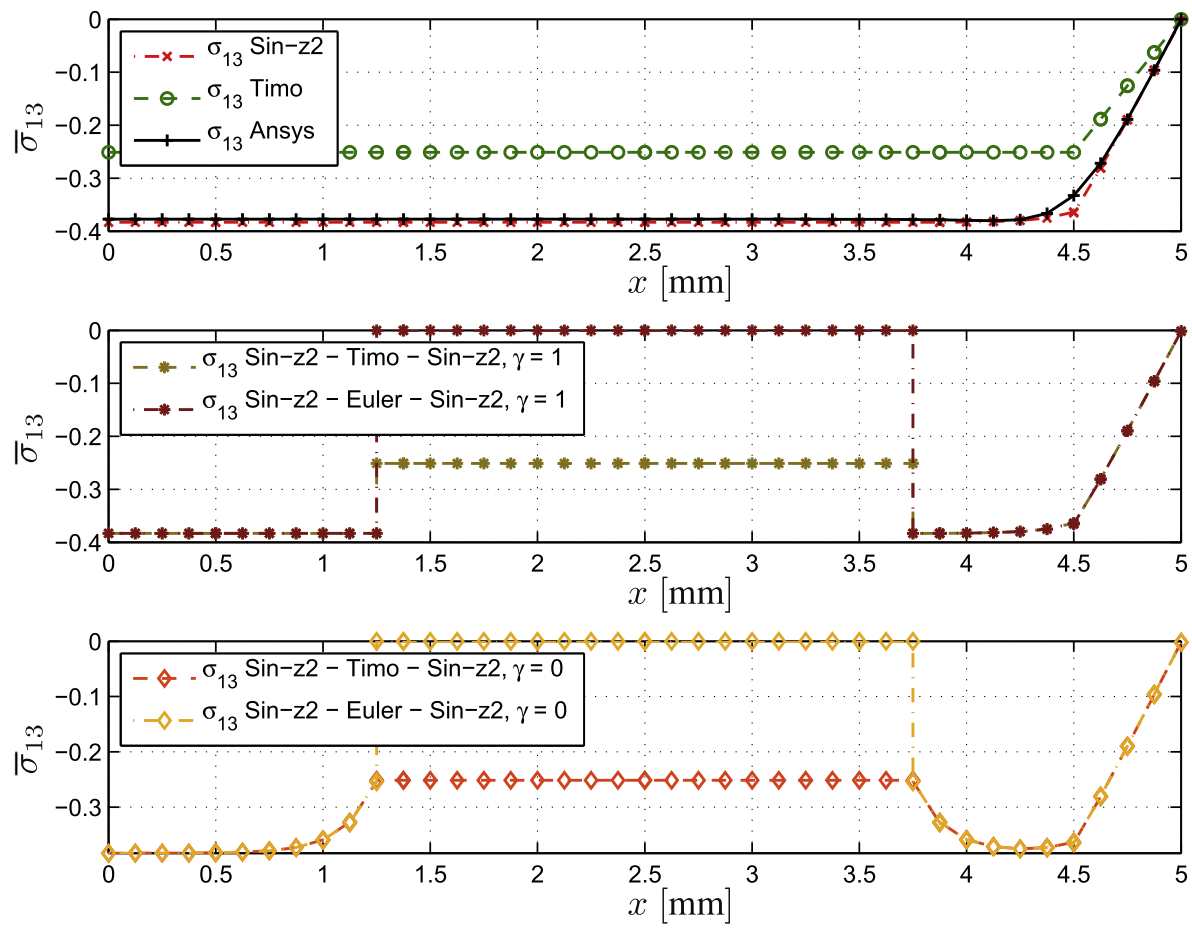

Fig. 12. Homogeneous Material: $\bar{\sigma}_{13}$ at $z=0$ for different kinematical models $(s=10)$.

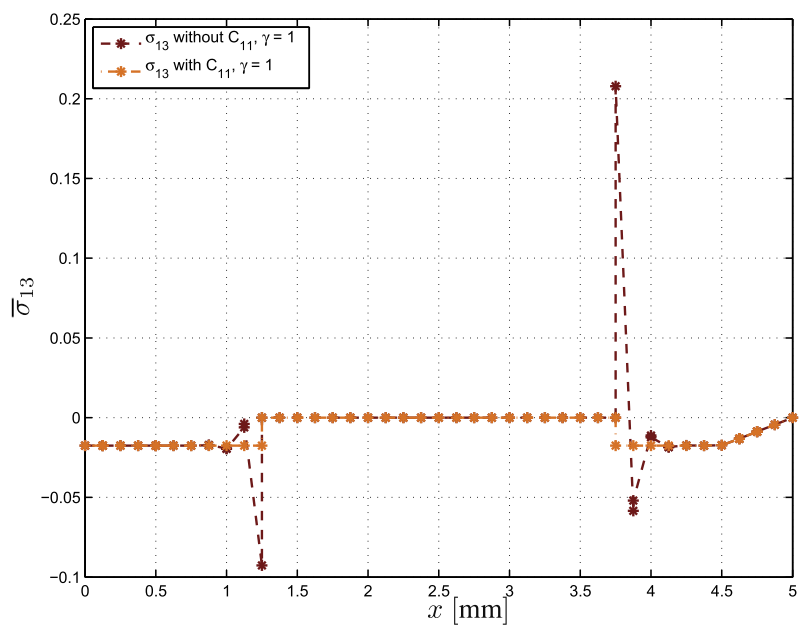

Fig. 13. Sandwich Material: $\bar{\sigma}_{13}$ over length for $\gamma=1 ; 1000 \cdot C_{11_{\text {core }}}=C_{11_{\text {face }}} ; s=10$.

one result with the Euler model is represented. We can observe from Fig. 15 that the use of XVF with $\gamma=0$ makes the variation of $\bar{\sigma}_{11}$ discontinuous at the interfaces while the results remain continuous for $\gamma=1$ and the influence of the localized pressure is also well captured. Again, a transition zone from the complex to the simple model for $\gamma=0$ can be observed for $\bar{\sigma}_{13}$. In the domain using the simple kinematics or in the domain using the Sin-z2 kinematic, but far from the interface, values are in good agreement with the monomodel results. For $\gamma=1$, the same abrupt change at the interfaces as for the homogeneous beam occurs, which allows to recover the distributions of the involved monomodel at any points of the structure.

In Fig. 17, the distributions of the stresses through the thickness at the middle of the beam and at the support show that the local response of the refined Sin-z2 model is preserved. It is also the case for $\gamma=0$, the end of the beam being not affected by the transition zone of the coupling.

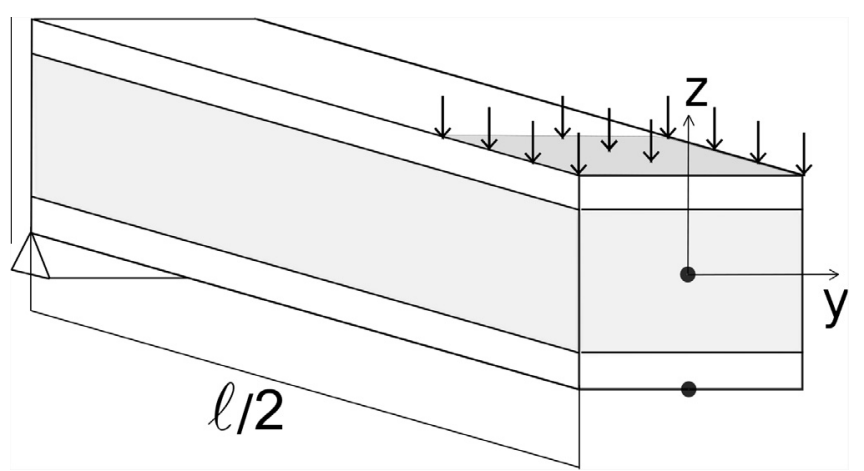

Fig. 14. Sandwich beam configuration with local pressure load.

As seen before, the coupling of different kinematics can induce a transition zone which must not disturb the results in the region of interest. Hence, the influence of the size of the complex zone is next studied for two cases $\gamma=0$ and $\gamma=1$. A regular mesh consisting of 20 elements is used for the half of the beam. By varying the number of elements in the central portion of the half-beam, the size of the simple and complex subregion is, therefore, varied. The number of elements in the central part of the beam corresponding to the simple kinematics is increased from 0 (Sinus $z^{2}$ monomodel) to 18 . For this latter, only one element at each end of the beam is associated with the refined kinematics. Figs. 18 and 19 represent the variation of the error rate on the maximal value of $\bar{\sigma}_{11}$ and $\bar{\sigma}_{13}$ with respect to the number of simple elements (i.e. the size of the central simple zone). This error rate is defined as $\Delta \sigma_{i j}=100 \frac{\bar{\sigma}_{i j}-\bar{\sigma}_{i j}^{\text {Sin-z2 }}}{\bar{\sigma}_{i j}^{\text {Sin-22 }}}$. It can be inferred from these figures that $\gamma=1$ yields better results, in particular for the transverse shear stress. Only one complex element can be used without affecting the quality of the results at the end of the beam. For $\gamma=0$, the size of the complex zone must be multiplied by four to achieve an error rate of $2 \%$. As far as the normal stress is concerned, a gain of 2 can be 

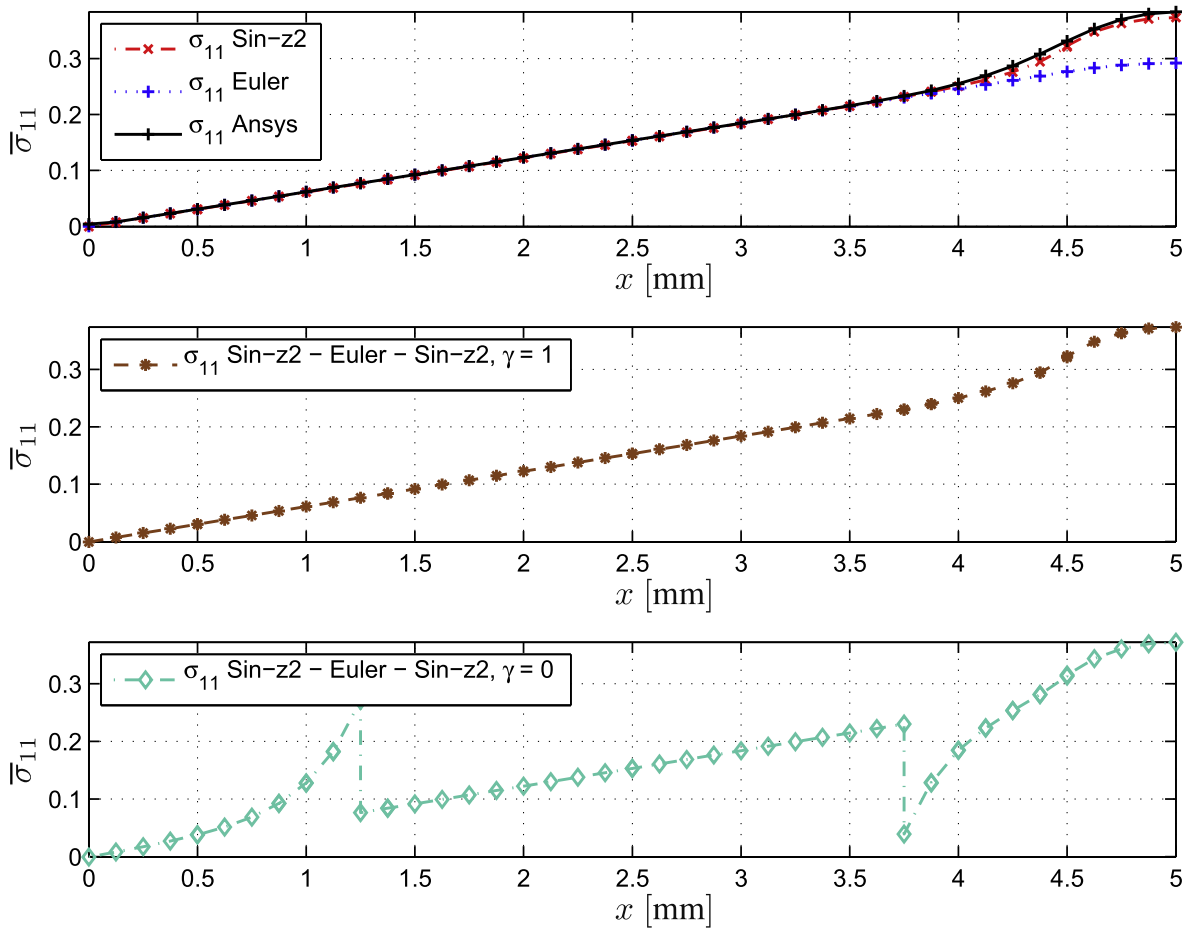

Fig. 15. Sandwich Material: $\bar{\sigma}_{11}$ at $z=-\frac{h}{2}$ with Euler combinations over beam length; $s=10$.
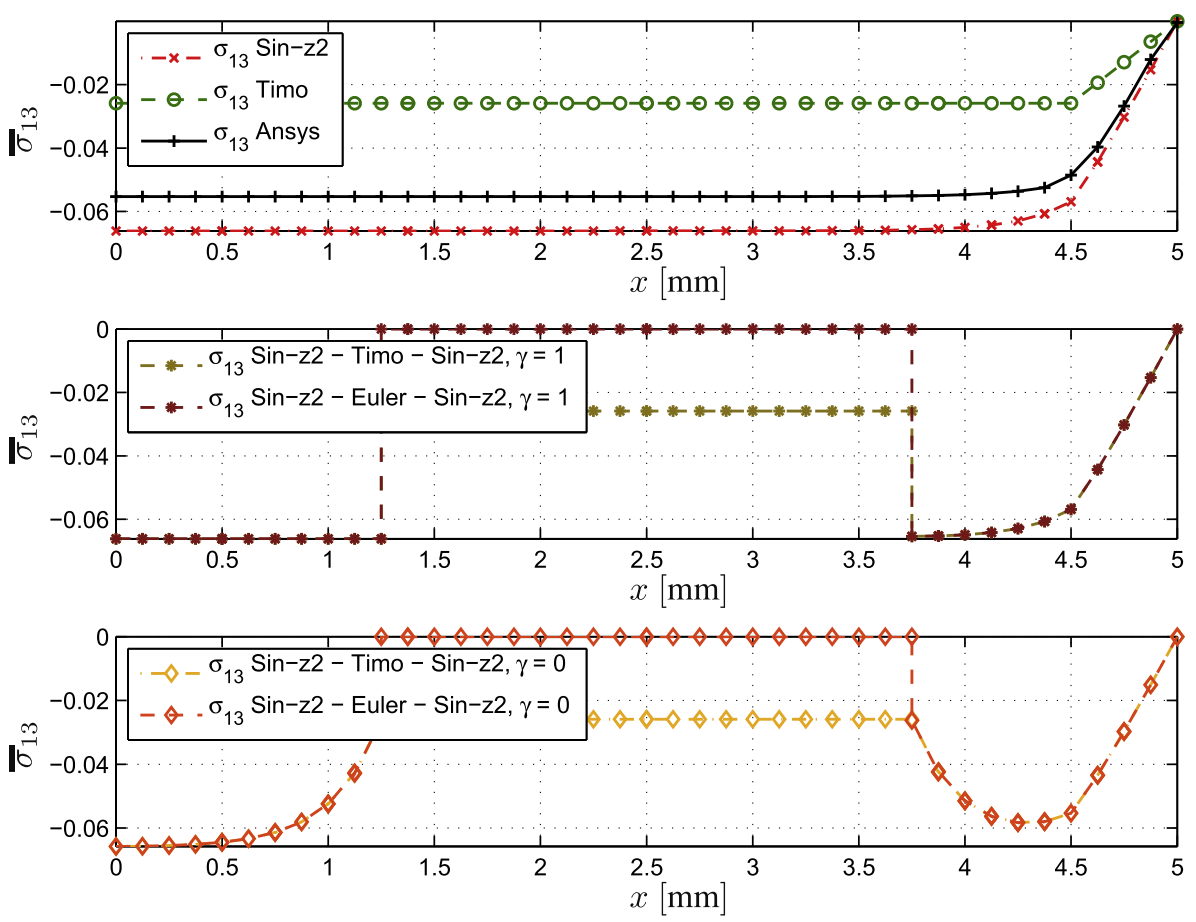

Fig. 16. Sandwich Material: $\bar{\sigma}_{13}$ at $z=0$ with Timoshenko and Euler combinations over beam length; $s=10$.

obtained. In contrast to the transverse shear stress, which is affected mainly by local effects, the in-plan stress is dominated by the global behavior of the whole model, here especially in terms of the deflection $u_{3}$. An unbalanced kinematically heterogenous model will not provide a smooth global behavior.

To illustrate the efficiency of the XVF approach with $\gamma=1$, the distribution of the transverse shear stress is shown in Fig. 20 with a mesh including 16 simple elements. We see clearly that the variation of the stress is perturbed for $0<x<\frac{l}{20}=\frac{h}{2}$ in the region of interest for $\gamma=0$. This is not the case for $\gamma=1$ where the results of the complex models are recovered in this region.

This attractive feature allows us to confine the richer model in a very small region where accurate stresses must be recovered. It can yield to a drastic reduction of the computational cost. 

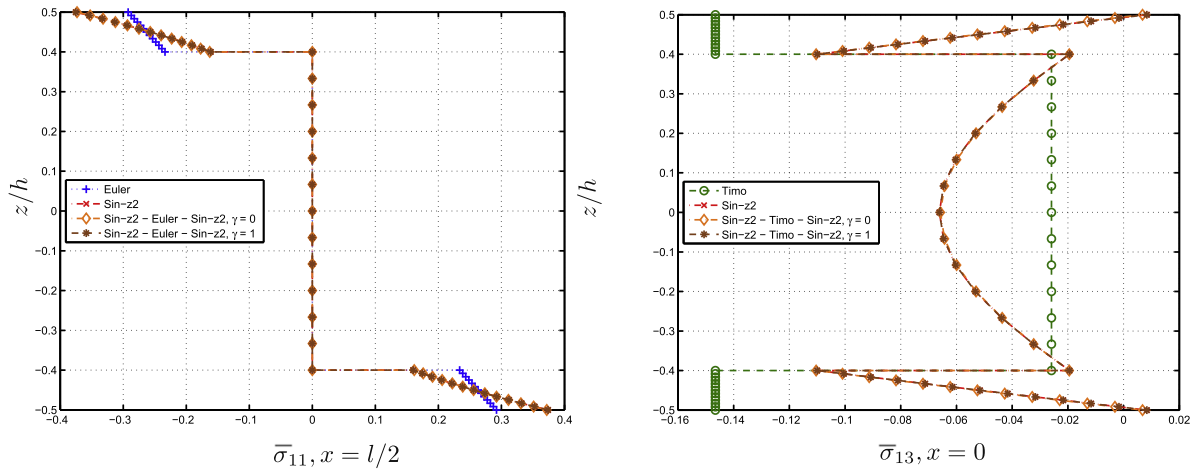

Fig. 17. Sandwich Material: (a): $\bar{\sigma}_{11}$ with Euler; (b): $\bar{\sigma}_{13}$ with Timoshenko; $s=10$.

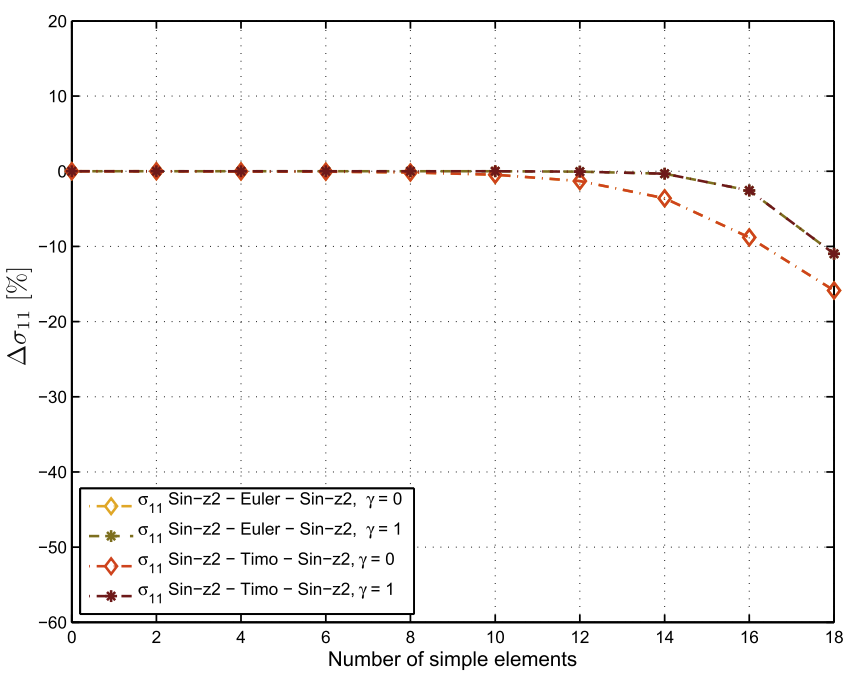

Fig. 18. Sandwich Material: error rate on $\bar{\sigma}_{11}$ for increasing number of simple elements; $s=10$.

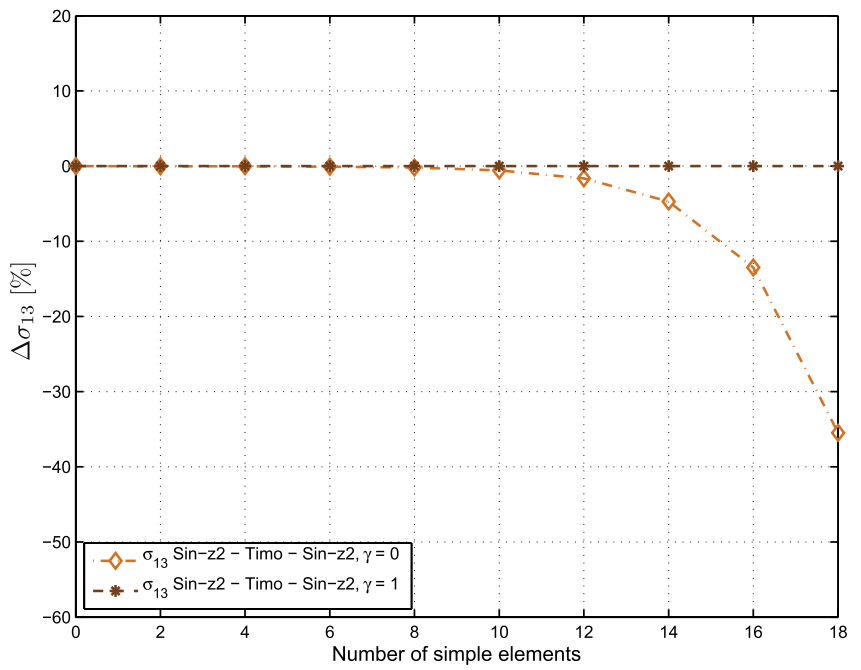

Fig. 19. Sandwich Material: Error rate on $\bar{\sigma}_{13}$ for increasing number of simple elements; $s=10$.
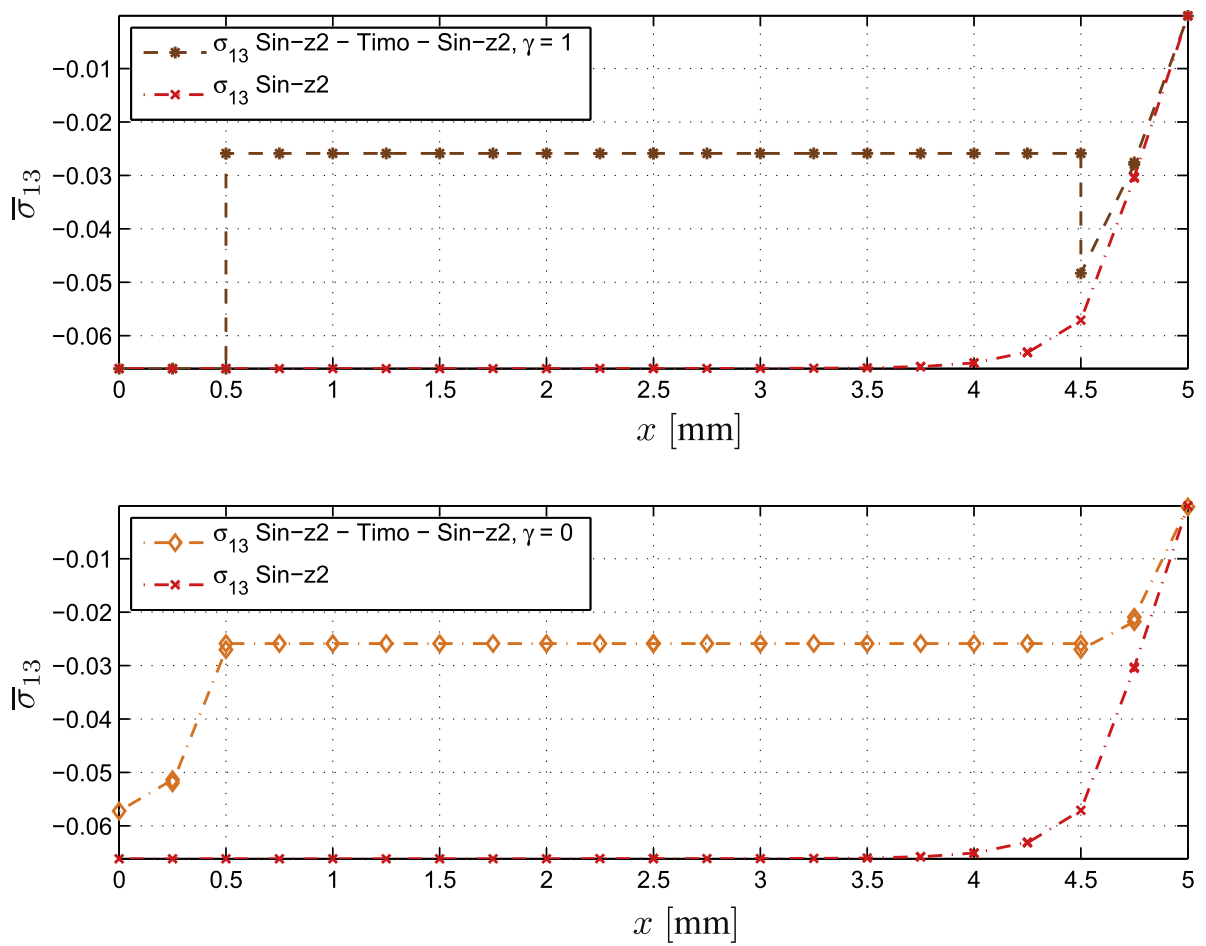

Fig. 20. Sandwich Material: $\bar{\sigma}_{13}$ over beam length with 16 Timoshenko theory elements; $s=10$. 


\section{Conclusions}

In this work, the XVF method is used to couple heterogeneous kinematics with homogeneous dimensionality to derive an efficient numerical tool for the modeling of composite beams. For that, the refined Sinus model including the transverse normal deformation is coupled with classical simple models (Euler-Bernoulli, Timoshenko). A new coupling operator is introduced to take into account the layered structures without decreasing the performance of the method. It has been analytically shown that two coupling schemes can be distinguished based on the values of the weighting scalar parameter $\gamma$. The presented results lead to following statements:

- the penalty technique is included in XVF (for $\gamma \in[0,1[$ );

- for $\gamma \in[0,1[$, the accuracy of the results is affected by the coupling in the vicinity of the interface, in particular in the complex zone;

- for $\gamma=1$, an abrupt change from the complex model level towards the simple model level is obtained without affecting the results of the refined model. It allows us to reduce the size of the complex zone;

- the stresses are accurate in the complex sub-domain (only far from the interface if $\gamma \in[0,1[)$ because they are only related to the gradient of the displacement, i.e., a local behavior; on the contrary, the global response (displacement) is in all case influenced by the coupling.

Therefore, the proposed approach allows us to decrease the computational cost for the analysis of composite structures by confining the refined and accurate models in the zone of interest. Further studies must be investigated. In particular, it would be interesting to extend the present approach to the coupling of ESL and LayerWise models: these latter, more expensive kinematics may be limited to a small region hosting important stress gradients (e.g., free-edge effects).

\section{Appendix A. Expressions of $F$}

The expressions of $\boldsymbol{F}_{\boldsymbol{\epsilon}}$ are given for the three models of the present work:

Euler-Bernoulli

$\boldsymbol{F}_{\epsilon}=\left[\begin{array}{ccccccccccc}1 & 0 & -z & 0 & 0 & 0 & 0 & 0 & 0 & 0 & 0 \\ 0 & 0 & 0 & 0 & 0 & 0 & 0 & 0 & 0 & 0 & 0 \\ 0 & 0 & 0 & 0 & 0 & 0 & 0 & 0 & 0 & 0 & 0\end{array}\right]$

Timoshenko

$\boldsymbol{F}_{\epsilon}=\left[\begin{array}{lllllllllll}1 & 0 & 0 & 0 & 0 & 0 & z & 0 & 0 & 0 & 0 \\ 0 & 1 & 0 & 0 & 0 & 1 & 0 & 0 & 0 & 0 & 0 \\ 0 & 0 & 0 & 0 & 0 & 0 & 0 & 0 & 0 & 0 & 0\end{array}\right]$

$\operatorname{Sin}-z 2$

$\boldsymbol{F}_{\epsilon}=\left[\begin{array}{ccccccccccc}1 & 0 & 0 & 0 & f(z)-z & 0 & f(z) & 0 & 0 & 0 & 0 \\ 0 & 1 & 0 & f^{\prime}(z)-1 & 0 & f^{\prime}(z) & 0 & 0 & z & 0 & z^{2} \\ 0 & 0 & 0 & 0 & 0 & 0 & 0 & 1 & 0 & 2 z & 0\end{array}\right]$

\section{Appendix B. Integration of the material behavior over the cross- section}

To compute the elementary stiffness matrices, it is needed to integrate the material behavior through the cross-section of the beam. It depends on the involved kinematics. In the following, only the dofs of each considered kinematics are kept in the expression of $\mathcal{E}_{\epsilon}$ (Eq. (34)) to compute $\boldsymbol{k}$. They are given for the three cases.

For Euler-Bernoulli

$\boldsymbol{k}=\int_{S}\left[\begin{array}{cc}\bar{C}_{11} & -z \bar{C}_{11} \\ s y m & z^{2} \bar{C}_{11}\end{array}\right] d S$

with $\mathcal{E}_{\epsilon}^{E B}=\left[v_{0}^{\prime} w_{0}^{\prime \prime}\right]^{T}$

For Timoshenko

$\boldsymbol{k}=\int_{S}\left[\begin{array}{cccc}\bar{C}_{11} & 0 & 0 & z \bar{C}_{11} \\ & \bar{C}_{55} & \bar{C}_{55} & 0 \\ & & \bar{C}_{55} & 0 \\ & \text { sym } & & z^{2} \bar{C}_{11}\end{array}\right] d S$

with $\mathcal{E}_{\epsilon}^{\text {Timo }}=\left[v_{0}^{\prime} w_{0}^{\prime} \theta \theta^{\prime}\right]^{T}$

For $\operatorname{Sin}-z 2$

$\boldsymbol{k}=\int_{S}\left[\begin{array}{ccccccc}\bar{C}_{11} & 0 & 0 & \bar{C}_{11}(f(z)-z) & 0 & \bar{C}_{11} f(z) & \ldots \\ & \bar{C}_{55} & \bar{C}_{55}\left(f^{\prime}(z)-1\right) & 0 & \bar{C}_{55} f^{\prime}(z) & 0 & \ldots \\ & & \bar{C}_{55}\left(f^{\prime}(z)-1\right)^{2} & 0 & \bar{C}_{55}\left(f^{\prime}(z)-1\right) f^{\prime}(z) & 0 & \ldots \\ & & & \bar{C}_{11}(f(z)-z)^{2} & 0 & \bar{C}_{11}(f(z)-z) f(z) & \ldots \\ & & & & \bar{C}_{55} f^{\prime}(z)^{2} & 0 & \ldots \\ & & & & & & \\ & & & & & & \ldots \\ & & & & & & \ldots \\ & & & & & & \ldots\end{array}\right.$

$\left.\begin{array}{lcccc}\ldots & \bar{C}_{13} & 0 & 2 \bar{C}_{13} z & 0 \\ \ldots & 0 & \bar{C}_{55} z & 0 & \bar{C}_{55} z^{2} \\ \ldots & 0 & \bar{C}_{55} z\left(f^{\prime}(z)-1\right) & 0 & \bar{C}_{55} z^{2}\left(f^{\prime}(z)-1\right) \\ \ldots & \bar{C}_{13}(f(z)-z) & 0 & 2 \bar{C}_{13} z(f(z)-z) & 0 \\ \ldots & 0 & \bar{C}_{55} z f^{\prime}(z) & 0 & \bar{C}_{55} z^{2} f^{\prime}(z) \\ \ldots & \bar{C}_{13} f(z) & 0 & 2 \bar{C}_{13} z f(z) & 0 \\ \ldots & \bar{C}_{33} & 0 & 2 \bar{C}_{33} z & 0 \\ \ldots & & \bar{C}_{55} z^{2} & 0 & \bar{C}_{55} z^{3} \\ \ldots & & & 4 \bar{C}_{33} z^{2} & 0 \\ \ldots & \text { sym } & & & \bar{C}_{55} z^{4}\end{array}\right] d S$

with $\mathcal{E}_{\epsilon}^{\text {Sin-z2 }}=\left[v_{0}^{\prime} w_{0}^{\prime} v_{1} v_{1}^{\prime} \theta \theta^{\prime} w_{1} w_{1}^{\prime} w_{2} w_{2}^{\prime}\right]^{T}$

\section{References}

[1] Simulia. ABAQUS Version 6.13: Analysis User's Manual, 2013.

[2] Fish J. The s-version of the finite element method. Comput Struct 1992;43(3): 539-47.

[3] Fish J, Markolefas S. The s-version of the finite element method for multilayer laminates. Int J Numer Methods Eng 1992;33:1081-105.

[4] Reddy JN, Robbins Jr DH. Theories and computational models for composite laminates. Appl Mech Rev 1994;47(6):6.

[5] Gendre L, Allix O, Gosselet P. A two-scale approximation of the Schur complement and its use for non-intrusive coupling. Int J Numer Methods Eng 2011;87(9):889-905.

[6] Ben Dhia H, Rateau G. The Arlequin method as a flexible engineering design tool. Int J Numer Methods Eng 2005;62:1442-62. 
[7] Hu H, Belouettar S, Potier-Ferry M, Daya El M. Multi-scale modelling of sandwich structures using the arlequin method part i: linear modelling. Finite Elem Anal Des 2009;45:37-51.

[8] Hu H, Belouettar S, Potier-Ferry M, Daya EM, Makradi A. Multi-scale nonlinear modelling of sandwich structures using the Arlequin method. Compos Struct 2010;92:515-22.

[9] Yu K, Hu H, Chen S, Belouettar S, Potier-Ferry M. Multi-scale techniques to nalyze instabilities in sandwich structures, Compos Struct 2013:96:751-62.

[10] Biscani F, Giunta G, Belouettar S, Carrera E, Hu H. Variable kinematic beam elements coupled via Arlequin method. Compos Struct 2011;93:697-708.

[11] Biscani F, Giunta G, Belouettar S, Carrera E, Hu H. Variable kinematic plate elements coupled via Arlequin method. Int J Numer Methods Eng 2012; 91:1264-90.

[12] Prager W. Variational principles for linear elastostatics for discontinuous displacements, strains and stresses. In: Broberg B, Hult J, Niordson F, editors. Recent progress in applied mechanics: the Folke Odqvist vol. Stockholm: Almqusit and Wiksell; 1967.

[13] Carrera E, Pagani A, Petrolo M. Use of Lagrange multipliers to combine 1D variable kinematic finite elements. Comput Struct 2013;129:194-206.

[14] Bernardi C, Maday Y, Patera AT. A new non conforming approach to domain decomposition; the mortar element method. In: Non linear partial differential equations and their applications. College de France seminar. London: Pitman; 1967.

[15] Aminpour M, Ransom J, Mc Cleary S. A coupled analysis method for structures with independently modeled finite element subdomains. Int J Numer Methods Eng 1995;38:3695-718.
[16] J.B. Ransom, On multifunctional collaborative methods in engineering science (PhD thesis), Langley Research Center, September 2001.

[17] Park KC, Felippa CA. A variational framework for solution method developments in structural mechanics. J Appl Mech 1998;65:242-9.

[18] Park KC, Felippa CA. A variational principle for the formulation of partitioned structural systems. Int J Numer Methods Eng 2000;47:395-418.

[19] Blanco PJ, Feijóo RA, Urquiza SA. A variational approach for coupling kinematically incompatible structural models. Comput Methods Appl Mech Eng 2008;197:1577-602.

[20] Kim H-G. Interface element method (IEM) for a partitioned system with non-matching interfaces. Comput Methods Appl Mech Eng 2002;191: 3165-94.

[21] Hetherington J, Rodríguez-Ferran A, Askes H. The bipenalty method for arbitrary multipoint constraints. Int J Numer Methods Eng 2013;93: 465-574.

[22] Polit O, Vidal P, D'Ottavio M. Robust $C^{0}$ high-order plate finite element for thin to very thick structures: mechanical and thermo-mechanical analysis. Int Numer Methods Eng 2012;40:429-51. http://dx.doi.org/10.1002/nme.3328.

[23] Vidal P, Polit O. A thermomechanical finite element for the analysis of rectangular laminated beams. Finite Elem Anal Des 2006(42):868-83.

[24] Vidal P, Polit O. A family of sinus finite elements for the analysis of rectangular laminated beams. Compos Struct 2008(84):56-72.

[25] Vidal P, Polit O. Assessment of the refined sinus model for the non-linear analysis of composite beams. Compos Struct 2009(87):370-81.

[26] H. Hu. Modélisation multi-échelle des structures sandwich ( $\mathrm{PhD}$ thesis) Université Paul Verlaine Metz, 2006. 